\title{
Economic Evaluation of Community-Based Falls Prevention Interventions: A Systematic Methodological Overview of Systematic Reviews
}

\author{
Mr Joseph Kwon ( $\square$ jkwon6@sheffield.ac.uk) \\ University of Sheffield, Regent Court (ScHARR) \\ Hazel Squires \\ University of Sheffield, Regent Court (ScHARR) \\ Matt Franklin \\ University of Sheffield, Regent Court (ScHARR) \\ Yujin Lee \\ University of Warwick \\ Tracey Young \\ University of Sheffield, Regent Court (ScHARR)
}

\section{Research Article}

Keywords: falls prevention, economic evaluation, systematic overview

Posted Date: November 23rd, 2021

DOI: https://doi.org/10.21203/rs.3.rs-481557/v2

License: @ (i) This work is licensed under a Creative Commons Attribution 4.0 International License. Read Full License 


\section{Abstract}

Background: Falls impose significant health and economic burdens on older people, making their prevention a priority for care decision-makers. The volume of falls prevention economic evaluations has increased, the findings from which have been synthesised by systematic reviews (SRs) with pre-specified criteria (e.g., objectives, eligibility, data extraction). Such SRs can inform commissioning and design of future evaluations, particularly decision models; however, their findings can be biased and partial dependent on their pre-specified criteria. This study aims to conduct a systematic overview (SO) to: (1) systematically identify SRs of community-based falls prevention economic evaluations; (2) describe the methodology and findings of SRs; (3) critically appraise the methodology of SRs; and (4) suggest commissioning recommendations based on SO findings.

Methods: The SO followed the PRISMA guideline and the Cochrane guideline on SO, covering the period 2003-2020. Identified SRs' aims, search strategies and results, extracted data fields, quality assessment methods and results, and commissioning and research recommendations were synthesised. The comprehensiveness of previous SRs' data synthesis was judged against criteria drawn from expert guideline and academic literature on falls prevention/public health economic evaluation. Outcomes of general population, lifetime decision models were re-analysed to inform commissioning recommendations. The SO protocol is registered in the Prospective Register of Systematic Reviews (CRD42021234379).

Results: Seven SRs were identified, which extracted 8 to 33 data fields from 44 relevant economic evaluations. Four economic evaluation methodological/reporting quality checklists were used; three SRs narratively synthesised methodological features to varying extent and focus. SRs generally did not appraise decision modelling features, including methods for characterising dynamic complexity of falls risk and intervention need. Their commissioning recommendations were based mainly on cost-per-unit ratios (e.g., incremental cost-effectiveness ratios) and neglected aggregate impact. There is model-based evidence of multifactorial and environmental interventions, home assessment and modification and Tai Chi being cost-effective but also the risk that they exacerbate social inequities of health.

Conclusions: Current SRs of falls prevention economic evaluations do not holistically inform commissioning and evaluation design. Accounting for broader decisional factors including intervention reach and capacity constraints and a broader grasp of methodological nuances of economic evaluations, particularly decision models, are needed.

\section{Background}

An ageing population with increased prevalence of falls in older age (e.g., aged 60+ years) has made falls prevention a global public health priority (1). Falls can cause mortality and substantial morbidity burden on older people including fear of falling (2), depression (3), functional decline (4), and fatality from serious injuries $(5)$ with high care system costs $(6,7)$ and wider societal burden (i.e., informal caregiver burden and declined social interaction) $(8,9)$.

Falls prevention interventions have been found to be effective in reducing the number of falls and fallers in community settings (10-12). Accordingly, costeffectiveness evidence from falls prevention economic evaluations has grown; the most recent Cochrane review of randomized controlled trials (RCTs) identified 12 economic evaluations for community-based falls prevention exercise alone (12).

The rising volume of economic evaluations has been accompanied by systematic reviews of available evidence. For a well-formulated research question, a systematic review uses systematic and explicit methods to identify relevant studies, synthesise relevant extracted data, and critically appraise their quality (13).

Two central functions of systematic reviews of economic evaluations can be: (A) to inform commissioning decisions; and (B) to summarise and evaluate the methodological features of economic evaluation in a topic area. Related to (A), the reviews can aid commissioning decisions by summarising the evaluation results most applicable to the decision-making context and/or identifying existing decision models that can be adapted and re-used (14). In England and Wales, the development of the National Institute for Health and Care Excellence's (NICE's) falls prevention guideline (version CG21, later updated as CG161 (15)) involved a systematic review of falls prevention economic evaluations (16).

Related to (B), systematic reviews can detail and critically appraise methodological features that significantly affect the evaluation results such as the identification, measurement and valuation of all relevant costs and consequences and structural assumptions made by decision models (14, 17). The appraisal could apply a pre-established checklist for methodological/reporting quality such as the Consolidated Health Economic Evaluation Reporting Standards (CHEERS) (18) and/or narratively synthesise methodological strengths and limitations. The findings from the methodological appraisal would also facilitate the conceptualisation of future economic evaluations, particularly decision models, and the identification of relevant data sources (14). Additionally, and related to both $(A)$ and $(B)$, such appraisal would enable commissioners to consider the wide range of methodological factors that may qualify the evaluation results before applying them to the decision problem.

A systematic overview uses explicit and systematic methods to identify previous systematic reviews in a topic area (19). It thus provides the highest level of economic evidence that can inform commissioning decisions as well as the opportunity for critically appraising the methodology of previous systematic reviews, specifically regarding how well they have performed the above functions (A) and (B). This would improve the methodological quality of: (i) future systematic reviews in the topic area; (ii) commissioning decisions based on the reviews; and (iii) future economic evaluations that utilise the reviews to conceptualise and implement their methodologies. The systematic overview is hence of interest both to consumers of economic evidence (i.e., commissioners, falls prevention professionals and patient groups) and to methodologists (i.e., systematic reviewers and falls prevention evaluators and modellers).

\section{Aim And Objectives}

The aim is to conduct a systematic overview of previous systematic reviews of community-based falls prevention interventions. The objectives are to: 
1. Systematically search for and identify previous systematic reviews of community-based falls prevention economic evaluations;

2. Describe the methods and findings of previous systematic reviews, including their aim, search strategy and results, data extracted, quality assessment and commissioning and research recommendations;

3. Critically appraise the methodology of previous systematic reviews and highlight areas of improvement for future systematic reviews;

4. Suggest commissioning recommendations for falls prevention interventions based on syntheses of results and methodological quality of economic evaluations identified by systematic overview.

\section{Methods}

The systematic overview followed the Cochrane guideline on overview of reviews (19) and the Preferred Reporting Items for Systematic Reviews and MetaAnalyse (PRISMA) guideline (13). See Supplementary material for the PRISMA checklist. The review protocol is registered in the Prospective Register of Systematic Reviews (CRD42021234379).

\section{Search strategy and selection criteria}

The search covered the period between January 2003 and December 2020 and 12 academic databases: Medline, Embase, PubMed, CDSR, CENTRAL, EconLit, CINAHL, PsycInfo, ASSIA, CRD, CEA Registry and PEDro. Grey literature studies were searched from online sites of Department of Health, Chartered Society of Physiotherapy, College of Occupational Therapy, Royal College of Nursing and Age UK. The start date was chosen based on a background knowledge that the number of economic evaluations before 2003 is low (16). The search strategy was an intersection between terms for falls, terms for older people and terms for economic evaluation. The Medline search strategy is given in the Supplementary material. References and citations of included studies were also searched.

Two researchers independently reviewed the titles and abstracts of identified articles at the first stage and the full texts of approved article at the second stage. Those that received two second-stage approvals were included for data extraction. Another researcher arbitrated in case of disagreement.

Included studies must have conducted a systematic review - i.e., involving the use of explicit, reproducible methodology, comprehensive search strategy and acceptable methods for data extraction and validity assessment of included studies by two or more researchers (19). Additionally, more than $50 \%$ of the review's included studies must have all of the following characteristics: (i) target population of community-dwelling older persons (aged 60+) and/or individuals aged 50-59 who are at high falls risk; (ii) any intervention designed to reduce the number of falls or fall-related injuries; (iii) any comparator(s); (iv) conduct full economic evaluations (i.e., comparative analyses of interventions in terms of their relative costs and consequences (17)); and (v) full text in English. Both single-vehicle evaluations (SVEs) (e.g., alongside RCTs) and decision models were included. Specific disease rehabilitation (e.g., for stroke) with minor falls prevention component were excluded.

\section{Data extraction and synthesis}

Following the Cochrane guideline (19), the following data were extracted from the included reviews and narratively synthesised: (1) author(s), publication year and review aim; (2) search strategy and results - period, databases, eligible study designs, eligible interventions, other eligibility criteria, and number of economic evaluations identified; (3) reference and characteristics of economic evaluations identified by reviews; (4) data fields extracted from economic evaluations by reviews; (5) methods for quality assessment of economic evaluations by reviews; and (6) commissioning and research recommendations made by reviews.

\section{Critical appraisal of previous systematic review methodology}

As recommended by the Cochrane guideline (19), the 11-item AMSTAR checklist (20) was applied independently by two reviewers to assess the reporting and methodological qualities of previous systematic reviews. Strengths and limitations stated by the systematic review authors were also noted.

The methodological quality of reviews was further critically appraised narratively. Specifically, the following guidelines and academic papers were used to establish what methodological features and outcomes of falls prevention economic evaluations should be extracted and analysed by the systematic reviews: (a) the expert guideline and checklist on conducting and reporting falls prevention economic evaluation (21); (b) the review of key methodological challenges to economic evaluation of geriatric public health interventions (22); (c) the health technology assessment checklist for quality assessment of decision models (23); and (d) the systematic methodological review of key methodological challenges to public health economic model development (24) and the associated model conceptualisation framework (14). Table 1 shows the data fields grouped into higher categories. 


\section{Category}

(A) Setting, population and evaluation framework

\section{Data field}

1. Bibliography: author(s); publication year

2. Setting and aim: country; region; decision-maker; evaluation aim

3. Study design (e.g., decision model)

4. Target population/sample demographics and comorbidities (e.g., residence ${ }^{1}$, age, sex, SES, health conditions unrelated to falls risk)

5. Type of analysis (e.g., CUA, CEA, CBA, ROI)

6. Perspective (e.g., public sector, societal)

7. Cost-effectiveness threshold clearly stated

8. Time horizon of analysis/model

9. Discount rates (if time horizon is longer than 1 year)

(B) Falls epidemiology

1. Target population/sample falls risk factors/profile at baseline

2. Fall type: definition; recording method

3. Health consequences of falls: injury type; long-term consequences (e.g., institutionalisation, excess mortality risk)

4. Health utility measurement: acute vs. long-term impact of falls on health utility; comorbidity-related impact on health utility

5. Economic consequences of falls ${ }^{2}$ : care resource types; unit costs; all-cause and fall-related costs ${ }^{3}$

6. Wider/societal consequences of falls (e.g., social isolation from fear of falling, informal caregiver burden, productivity loss)

(C) Falls prevention intervention

1. Intervention characteristics: type ${ }^{4}$; reach ${ }^{5}$; primary vs. secondary prevention; main components; staff type; duration, frequency and dose; mutual exclusivity ${ }^{6}$; comparator(s)

2. Intervention pathway: type (e.g., reactive, proactive, self-referred ${ }^{7}$ ); recruitment method; falls risk identification method; mutual exclusivity ${ }^{6}$

3. Intervention resource use (e.g., staff labour and training, transport, overheads)

4. Intervention costs ${ }^{2}$ : variable vs. fixed costs; economies of scale; societal costs (e.g., time opportunity cost, private co-payment)

5. Intervention implementation: uptake rate; adherence rate; sustainability rate

6. Intervention efficacy: match with incidence metric ${ }^{8}$; efficacy fall type $^{9}$; efficacy durability ${ }^{10}$; wider health benefits $^{11}$; intervention side effects

7. Intervention study characteristics: study design (e.g., RCT, meta-analysis); population/sample characteristics ${ }^{12}$

(D) Decision model features

1. Model type and justification of type

2. Model cycle length and justification of length

3. Methods for adopting a long-term model horizon ${ }^{13}$

4. Methods for characterising baseline demographics and falls risk of model target population ${ }^{14}$

5. Methods for characterising multiple falls in a year (recurrent falls) ${ }^{14}$

6. Methods for characterising dynamic progression of falls risk factors, long-term consequences of falls and falls prevention intervention need ${ }^{14,15}$

7. Methods for characterising dynamic progression in comorbidities and changes in care costs, mortality risks, institutionalisation risks and health utilities ${ }^{14}$

8. Methods for incorporating psychological and sociological variables as determinants of falls risk, falls prevention access and model outcomes ${ }^{14}$

9. Methods for incorporating budget and capacity constraints

10. Methods for reducing structural uncertainty of model prospectively ${ }^{16}$

11. Model validation methods/results: face; internal; external 


\begin{tabular}{|c|c|}
\hline Category & Data field \\
\hline \multirow{8}{*}{$\begin{array}{l}\text { (E) Evaluation methods and } \\
\text { results }\end{array}$} & 1. Cost-per-unit ratios (e.g., incremental cost per QALY gain) \\
\hline & 2. Aggregate health and cost outcomes (e.g., total intervention cost, total QALY gain, total number of falls prevented) \\
\hline & 3. Currency: original type/year; conversion to same currency for comparison \\
\hline & 4. Handling heterogeneity: subgroup analyses; targeting analyses (under budget or capacity constraint) \\
\hline & 5. Handling parameter uncertainty: deterministic sensitivity analysis; probabilistic sensitivity analysis \\
\hline & $\begin{array}{l}\text { 6. Scenario analyses: testing structural assumptions; scenario suggestions by stakeholders/decision-maker; value of } \\
\text { implementation analysis }{ }^{17}\end{array}$ \\
\hline & $\begin{array}{l}\text { 7. Equity analyses: intervention impact on social inequities in health; estimating efficiency cost of prioritising } \\
\text { vulnerable groups }{ }^{18}\end{array}$ \\
\hline & 8. Model cross-validity: comparison of results to previous models \\
\hline \multirow{2}{*}{$\begin{array}{l}\text { (F) Discussions by evaluation } \\
\text { authors }\end{array}$} & 1. Discussion on issues of generalisability and policy implementation \\
\hline & 2. Discussion on strengths and limitations of evaluation \\
\hline
\end{tabular}

Abbreviation: CBA: cost-benefit analysis; CEA: cost-effectiveness analysis; CUA: cost-utility analysis; QALY: quality-adjusted life year; RCT: randomised controlled trial; ROI: return on investment; SES: socioeconomic status

${ }^{1}$ Community-living or institutionalised.

${ }^{2}$ All costs and outcomes should be appropriately identified, measured and valued.

${ }^{3}$ Expert guideline on falls prevention economic evaluation recommends that evaluations report all-cause/total healthcare costs in the base case and fallrelated costs in sensitivity analysis $(21)$.

${ }^{4}$ Intervention type classification should follow the Prevention of Falls Network Europe categories (25).

5 Intervention reach refers to the number/proportion of persons in the target population accessing the intervention. It is a function of intervention's normative reach defined by its eligibility criteria and its implementation reach determined by implementation level (e.g., uptake rates) within the eligible population.

${ }^{6}$ Several intervention types and pathways can be non-mutually exclusive in a setting: e.g., reactive home assessment and modification for fallers discharged from hospitals and self-referred exercise.

${ }^{7}$ Reactive pathway is accessed immediately after a fall requiring medical attention. Proactive pathway is accessed via referrals by care professionals in the community. Self-referred pathway is accessed voluntarily by older persons based on community/peer marketing.

8 This only concerns decision models that import falls efficacy evidence from external intervention studies. Main falls incidence metrics are falls risk and falls rate, and their matching efficacy metrics are relative risk (RR) and rate ratio (RaR), respectively. Models should ensure that the external efficacy metric matches the internal falls incidence metric.

${ }^{9}$ Like note 8, this again only concerns decision models using external efficacy evidence. The fall type (e.g., hospitalised fall, fall-induced fracture) for the efficacy data should match that for the model incidence.

${ }^{10}$ Durability of intervention efficacy should not extend beyond the timespan of the intervention study unless the intervention receipt is sustained (21).

${ }^{11}$ For example, falls prevention exercise can have additional health benefits, e.g., reducing cardiovascular disease risk.

12 Decision models should ensure that the characteristics of the external intervention study's target population/sample (e.g., inclusion/exclusion criteria) match those of the model population.

${ }^{13}$ Lifetime horizon is recommended by the expert guideline on falls prevention economic evaluation (21).

${ }^{14}$ Reviews should note the modelling methods and the data type, source and quality reported by evaluations.

${ }^{15}$ An example of a method used to characterise the dynamic complexity of falls risk is to incorporate tunnel states in Markov cohort models to capture the secular age-related increase in falls risk (26). Long-term consequences of falls can be captured by incorporating the relationship between falls and a broader health profile such as frailty. Intervention need (i.e., eligibility) can change over time as individuals' falls risk profiles change.

${ }^{16}$ Prospective reduction in structural uncertainty can be achieved through stakeholder engagement and model conceptualisation that precedes model parameterisation (14).

17 This aims to assess the impact of implementation improvements (e.g., higher uptake) on economic outcomes (27).

${ }^{18}$ Efficiency cost may arise if prioritising intervention at a particular vulnerable group for an equity objective results in efficiency loss (i.e., there is an equity-efficiency trade-off) (28).

\section{Commissioning recommendation by this systematic overview}

The results and methodological features were extracted from a subset of primary economic evaluations and re-analysed to inform the commissioning recommendations made by the systematic overview. Specifically, data were extracted from general population models (as opposed to models targeting specific patient groups) analysed over lifetime horizons since these are most informative for jurisdiction-level commissioning decisions on falls prevention 
$(21,29)$. Such re-analysis of primary study outcomes is recommended by the Cochrane guideline if this suits the purpose of the systematic overview $(19)$. Key methodological features of the models that are likely to influence their outcomes are considered while formulating the commissioning recommendations.

\section{Results}

\section{Systematic overview search results}

Figure 1 presents the PRISMA flow diagram: 15,730 titles and abstracts were screened; and 55 full texts screened, from which 7 systematic reviews were identified.

\section{Methods and findings of previous systematic reviews Aim, search strategy and search result}

Table 2 summarises the aim, search strategy and search results of previous systematic reviews. The reviews shared the aim of assessing the costeffectiveness evidence within their targeted intervention area. Two reviews specifically targeted community-based falls prevention interventions (30, 31); three targeted falls prevention in both community and institutionalised settings $(16,32,33)$; and two targeted a broader range of geriatric public health interventions, more than $50 \%$ of which were community-based falls prevention interventions $(34,35)$. Several reviews had further aims of informing: the development of the NICE falls prevention clinical guideline (16); the development of a new falls prevention decision model (31); the practice of and research on falls prevention exercise (33); and the methodologies of subsequent falls prevention economic evaluations $(32,34,35)$. All searches covered at least 4 academic databases, while three further covered grey literature sites. 
Table 2

Aim, search strategy and search results of previous systematic reviews of community-based falls prevention economic evaluations

\begin{tabular}{|c|c|c|c|c|c|}
\hline \multirow[t]{2}{*}{ Review } & \multirow[t]{2}{*}{ Aim } & \multicolumn{3}{|c|}{ Search strategy } & \multirow[t]{2}{*}{ Search results } \\
\hline & & $\begin{array}{l}\text { Coverage } \\
\text { period }\end{array}$ & Source & Target intervention/setting & \\
\hline \multirow[t]{2}{*}{$\begin{array}{l}\mathrm{RCN} \\
\text { review } \\
(16)\end{array}$} & $\begin{array}{l}\text { (1) Assess cost-effectiveness of falls } \\
\text { prevention interventions (any setting) }\end{array}$ & \multirow{2}{*}{$\begin{array}{l}\text { Database } \\
\text { inception } \\
\text { to April } \\
2003\end{array}$} & \multirow[t]{2}{*}{$\begin{array}{l}4 \text { academic } \\
\text { databases }\end{array}$} & \multirow[t]{2}{*}{$\begin{array}{l}\text { Falls prevention interventions in } \\
\text { community and extended care }\end{array}$} & \multirow{2}{*}{$\begin{array}{l}\text { Total: } 6 \\
\text { studies, of } \\
\text { which } 5 \text { CB } \\
\text { Models: } 1 \text { CB }\end{array}$} \\
\hline & $\begin{array}{l}\text { (2) Inform the NICE clinical guideline on falls } \\
\text { prevention for older people }\end{array}$ & & & & \\
\hline \multirow[t]{2}{*}{$\begin{array}{l}\text { Davis } \\
\text { review } \\
(30)\end{array}$} & \multirow[t]{2}{*}{$\begin{array}{l}\text { (1) Assess cost-effectiveness of community- } \\
\text { based falls prevention interventions }\end{array}$} & \multirow{2}{*}{$\begin{array}{l}\text { Database } \\
\text { inception } \\
\text { to July } \\
2008\end{array}$} & \multirow[t]{2}{*}{$\begin{array}{l}4 \text { academic } \\
\text { databases }\end{array}$} & \multirow[t]{2}{*}{$\begin{array}{l}\text { Community-based falls prevention } \\
\text { interventions }\end{array}$} & $\begin{array}{l}\text { Total: } 9 \\
\text { studies }\end{array}$ \\
\hline & & & & & Models: 3 \\
\hline \multirow[t]{3}{*}{$\begin{array}{l}\text { DJ } \\
\text { review } \\
(34)\end{array}$} & $\begin{array}{l}\text { (1) Assess cost-effectiveness of public health } \\
\text { interventions for older people (any setting) }\end{array}$ & \multirow[t]{3}{*}{$\begin{array}{l}2000 \text { to } \\
\text { July } 2015\end{array}$} & \multirow{3}{*}{$\begin{array}{l}5 \text { academic } \\
\text { databases and } \\
23 \text { grey } \\
\text { literature sites }\end{array}$} & \multirow{3}{*}{$\begin{array}{l}\text { Health promotion and primary prevention } \\
\text { interventions (except vaccination) for } \\
\text { older people in community and extended } \\
\text { care }\end{array}$} & \multirow{2}{*}{$\begin{array}{l}\text { Total: } 29 \\
\text { studies, of } \\
\text { which } 22 \text { CB } \\
\text { falls } \\
\text { prevention }\end{array}$} \\
\hline & \multirow{2}{*}{$\begin{array}{l}\text { (2) Evaluate methodological features and } \\
\text { quality of falls prevention economic } \\
\text { evaluations }\end{array}$} & & & & \\
\hline & & & & & $\begin{array}{l}\text { Models: } 10 \mathrm{CB} \\
\text { falls } \\
\text { prevention }\end{array}$ \\
\hline \multirow{2}{*}{$\begin{array}{l}\text { PHE } \\
\text { review } \\
(31)\end{array}$} & $\begin{array}{l}\text { (1) Assess cost-effectiveness of community- } \\
\text { based falls prevention interventions }\end{array}$ & \multirow{2}{*}{$\begin{array}{l}2003 \text { to } \\
\text { December } \\
2016\end{array}$} & \multirow{2}{*}{$\begin{array}{l}13 \text { academic } \\
\text { databases and } \\
7 \text { grey } \\
\text { literature sites }\end{array}$} & \multirow{2}{*}{$\begin{array}{l}\text { Community-based falls prevention } \\
\text { interventions recommended by } 2013 \text { NICE } \\
\text { guideline (CG161) }(15)^{2}\end{array}$} & $\begin{array}{l}\text { Total: } 26 \\
\text { studies }\end{array}$ \\
\hline & $\begin{array}{l}\text { (2) Inform development of falls prevention } \\
\text { economic model for English community } \\
\text { setting }\end{array}$ & & & & Models: 12 \\
\hline \multirow[t]{2}{*}{$\begin{array}{l}\text { Olij } \\
\text { review } \\
(32)\end{array}$} & $\begin{array}{l}\text { (1) Assess cost-effectiveness of falls } \\
\text { prevention interventions (any setting) }\end{array}$ & \multirow{2}{*}{$\begin{array}{l}\text { Database } \\
\text { inception } \\
\text { to May } \\
2017\end{array}$} & \multirow[t]{2}{*}{$\begin{array}{l}6 \text { academic } \\
\text { databases and } \\
\text { Google Scholar }\end{array}$} & \multirow[t]{2}{*}{$\begin{array}{l}\text { Falls prevention interventions in } \\
\text { community and extended care }\end{array}$} & \multirow{2}{*}{$\begin{array}{l}\text { Total: } 31 \\
\text { studies, of } \\
\text { which } 28 \mathrm{CB} \\
\text { Models: } 10 \mathrm{CB}\end{array}$} \\
\hline & $\begin{array}{l}\text { (2) Evaluate methodological features and } \\
\text { quality of falls prevention economic } \\
\text { evaluations }\end{array}$ & & & & \\
\hline \multirow[t]{2}{*}{$\begin{array}{l}\text { Huter } \\
\text { review } \\
(35)\end{array}$} & \multirow[t]{2}{*}{$\begin{array}{l}\text { (1) Evaluate how economic evaluations of } \\
\text { public health interventions for older people } \\
\text { (any setting) handled key methodological } \\
\text { challenges }^{1}\end{array}$} & \multirow[t]{2}{*}{$\begin{array}{l}2000 \text { to } \\
\text { March } \\
2018\end{array}$} & \multirow[t]{2}{*}{$\begin{array}{l}5 \text { academic } \\
\text { databases and } \\
23 \text { grey } \\
\text { literature sites }\end{array}$} & \multirow[t]{2}{*}{$\begin{array}{l}\text { Health promotion and primary prevention } \\
\text { interventions (except vaccination) for } \\
\text { older people in community and extended } \\
\text { care }\end{array}$} & $\begin{array}{l}\text { Total: } 37 \\
\text { studies, of } \\
\text { which } 25 \mathrm{CB} \\
\text { falls } \\
\text { prevention }\end{array}$ \\
\hline & & & & & $\begin{array}{l}\text { Models: } 11 \mathrm{CB} \\
\text { falls } \\
\text { prevention }\end{array}$ \\
\hline \multirow[t]{2}{*}{$\begin{array}{l}\text { Winser } \\
\text { review } \\
(33)\end{array}$} & $\begin{array}{l}\text { (1) Assess cost-effectiveness of exercise- } \\
\text { based falls prevention interventions (any } \\
\text { setting) }\end{array}$ & \multirow{2}{*}{$\begin{array}{l}\text { Database } \\
\text { inception } \\
\text { to } \\
\text { February } \\
2019\end{array}$} & $\begin{array}{l}6 \text { academic } \\
\text { databases }\end{array}$ & $\begin{array}{l}\text { Exercise-based falls prevention } \\
\text { interventions evaluated by RCTs in } \\
\text { community and extended care }\end{array}$ & $\begin{array}{l}\text { Total: } 12 \\
\text { studies, all CB } \\
\text { falls } \\
\text { prevention }\end{array}$ \\
\hline & $\begin{array}{l}\text { (2) Evaluate implications for clinical practice } \\
\text { and future research on falls prevention } \\
\text { exercise dosage }\end{array}$ & & & & Models: $1^{3}$ \\
\hline $\begin{array}{l}\text { grev } \\
\text { al c }\end{array}$ & $\begin{array}{l}\text { n: CB: community-based; DJ: Dubas-Jakobc } \\
\text { ge of Nursing; RCT: randomized controlled tr }\end{array}$ & CE: Natio & Istitute for $\mathrm{He}$ & and Care Excellence; PHE: Public Health & and; RCN: \\
\hline $\begin{array}{l}1 \text { These } \\
\text { unrelate }\end{array}$ & $\begin{array}{l}\text { : (i) measurement and valuation of informal c } \\
\text { ost in added life years; and (iv) accounting fo }\end{array}$ & $\begin{array}{l}\text { yiving; (ii) ac } \\
\text { ler non-healt }\end{array}$ & $\begin{array}{l}\text { unting for produ } \\
\text { effects of interve }\end{array}$ & $\begin{array}{l}\text { tivity costs (including unpaid work); (iii) acc } \\
\text { tions. }\end{array}$ & ting for \\
\hline 2 This $\mathrm{e}$ & les interventions such as vitamin $D$, hip prot & s and cogr & e behavioural th & apy (31). & \\
\hline
\end{tabular}

Overall, the reviews identified 44 economic evaluations of community-based falls prevention interventions, of which 21 were decision models. The recent decade has seen a significant increase, rising from 9 evaluations identified by the Davis review (30) in 2003 to 26 identified by the PHE review in 2017 (31). Table B in Supplementary material provides the reference and characteristics of identified economic evaluations, including their target population, type(s) of analysis, perspective(s), analysis time horizon, intervention(s) and comparator(s). No evaluation was identified by all seven reviews.

\section{Data fields extracted by systematic reviews}

Table 3 shows the data fields extracted from economic evaluations by previous reviews. There was a marked variation across reviews in the number of data fields extracted, ranging from 8 to 33. Data fields for model features were the most limited, restricted to model type and evidence source. No review quantitatively pooled the evaluation outcomes due to significant underlying methodological differences. 
Table 3

Data fields extracted by previous systematic reviews of community-based falls prevention economic evaluations. 1

Data fields

\section{Systematic reviews}

RCN (16) Davis (30) DJ (34) PHE (31) Olij (32) Huter (35) Winser (33)

(A) Setting, population and evaluation framework

Author(s) and publication year

Country/region

Study design (e.g., model, RCT)

TP/sample residence

TP/sample age and sex

Type of analysis (e.g., CUA)

Perspective (e.g., societal)

Time horizon/Follow-up period

Discount rates

Number of fields

ए \

[ ?

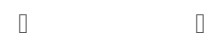

ㄴ ?

प

(B) Falls epidemiology

TP/sample falls risk factor(s)

Baseline falls risk estimates

Main health event (e.g., fall type)

Health utility instrument

Wider (e.g., non-health) outcomes

Health and social care consequence types

Societal consequence types

All-cause/comorbidity costs

Cost measurement method in RCT

Number of fields

(C) Falls prevention intervention

Intervention type

प

Primary vs. secondary prevention

Intervention components

Intervention duration

[

Q

प

प

\section{[}

[

Exercise intervention dosage

Professional staff involved

Comparator

Participant recruitment method/setting

Falls risk identification method

Intervention resource use

Intervention cost

Societal intervention resource/cost

Intervention fall-related efficacy

Intervention study sample size

Number of fields

1

(D) Decision model features

Model type

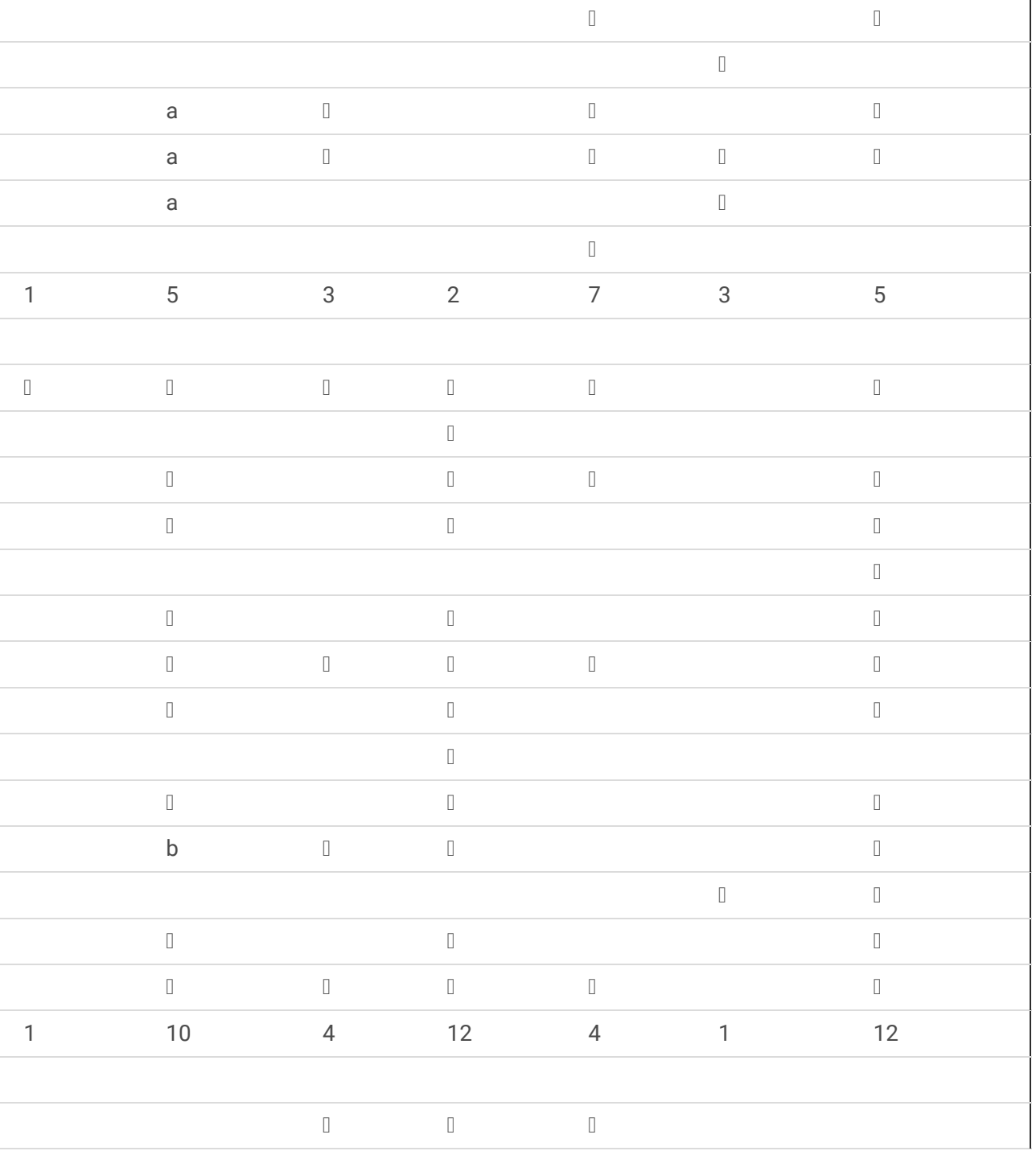




\begin{tabular}{|c|c|c|c|c|c|c|c|}
\hline \multirow[t]{2}{*}{ Data fields } & \multicolumn{7}{|c|}{ Systematic reviews } \\
\hline & $\mathrm{RCN}(16)$ & Davis (30) & DJ (34) & PHE (31) & Olij (32) & Huter (35) & Winser (33) \\
\hline \multicolumn{3}{|l|}{ Model data sources } & प & $\square$ & & & \\
\hline \multicolumn{5}{|l|}{ Characterising baseline falls risk estimates } & \multicolumn{3}{|l|}{ प } \\
\hline Number of fields & 0 & 0 & 2 & 2 & 2 & 0 & 0 \\
\hline \multicolumn{8}{|l|}{ (E) Evaluation methods and results } \\
\hline Cost-per-unit ratio (e.g., ICER) & ] & ] & प & $\square$ & ( & & ( \\
\hline \multicolumn{2}{|l|}{ Aggregate cost and health outcomes ${ }^{2}$} & [ & & ] & & & ( \\
\hline \multicolumn{2}{|l|}{ Original currency type } & 口 & प & & & & ( \\
\hline \multicolumn{2}{|l|}{ Converted results into same currency } & [ & प & & प & & \\
\hline \multicolumn{2}{|l|}{ Subgroup/targeting methods/results } & प & & $\square$ & प & & ( \\
\hline \multicolumn{2}{|l|}{ Handling parameter uncertainty ${ }^{3}$} & ( & & ( & 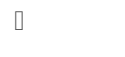 & & ( \\
\hline \multicolumn{4}{|l|}{ Scenario analysis methods/results ${ }^{4}$} & $\square$ & & & \\
\hline \multicolumn{4}{|l|}{ Equity analysis methods/results ${ }^{5}$} & $\square$ & & & \\
\hline Number of fields & 1 & 6 & 3 & 6 & 4 & 0 & 5 \\
\hline \multicolumn{8}{|l|}{ (F) Discussions by evaluation authors ${ }^{6}$} \\
\hline \multicolumn{3}{|l|}{ Generalisability and policy implementation } & $\square$ & $\square$ & & & \\
\hline \multicolumn{3}{|l|}{ Strengths and limitations } & ( & $\square$ & & प & \\
\hline Number of fields & 0 & 0 & 2 & 2 & 0 & 1 & 0 \\
\hline Total number of fields & 8 & 30 & 21 & 33 & 26 & 8 & 31 \\
\hline \multicolumn{8}{|c|}{$\begin{array}{l}\text { Abbreviation: CUA: cost-utility analysis; DJ: Dubas-Jakobczyk; ICER: incremental cost-effectiveness ratio; PHE: } \\
\text { of Nursing; RCT: randomized controlled trial; TP: target population }\end{array}$} \\
\hline \multicolumn{8}{|c|}{${ }^{1}$ This table does not account for data fields extracted by reviews for applying a quality assessment checklist. } \\
\hline \multicolumn{8}{|c|}{2 Includes outcomes such as total intervention cost and total number of falls prevented. } \\
\hline \multicolumn{8}{|c|}{3 Includes one-/two-way deterministic sensitivity analysis and probabilistic sensitivity analysis. } \\
\hline \multicolumn{8}{|c|}{${ }^{4}$ Analysis of alternative modelling assumptions: e.g., whether fear of falling exerts a health utility decrement. } \\
\hline \multicolumn{8}{|c|}{${ }^{5}$ Analysis of intervention impact on social inequalities/inequities in health. } \\
\hline \multicolumn{8}{|c|}{${ }^{6}$ These concern issues stated and discussed by the economic evaluation authors, not systematic reviewers. } \\
\hline \multicolumn{8}{|c|}{$\begin{array}{l}\text { a Distinguished between fall-related and all-cause care cost and reported detailed list: emergency department; hospitalization; outpatient visit; GP visit; } \\
\text { district nurse visit; home care; equipment; meal-on-wheel; day care centre; residential care; nursing home; patient and caregiver's cost (out-of-pocket } \\
\text { expenditure, time cost). }\end{array}$} \\
\hline
\end{tabular}

\section{Quality assessment of economic evaluations by systematic reviews}

All reviews except RCN applied a checklist to assess the reporting and methodological quality of their included studies. In total, four checklists were applied, all of them generic (i.e., all disease areas) and all-design (i.e., SVEs and models). Table $\mathrm{C}$ in Supplementary material lists the items of the checklists used, and Table 4 shows the quantitative checklist scores given to individual economic evaluations by the reviews. The scores are converted to percentage to ease comparison.

Table 4 Results of quality assessment by previous systematic reviews of community-based falls prevention economic evaluations 


\begin{tabular}{|c|c|c|c|c|c|c|c|c|c|c|}
\hline \multirow[t]{3}{*}{ \# } & \multirow[t]{3}{*}{ Primary study } & \multirow{3}{*}{$\begin{array}{l}\text { Systematic } \\
\text { review }^{1}\end{array}$} & \multicolumn{8}{|c|}{ Quality assessment results by checklist ${ }^{2}$} \\
\hline & & & $\begin{array}{l}\text { Davis } \\
\text { Drummond (17) }\end{array}$ & $\begin{array}{l}\text { DJ/Huter } \\
\text { Drummond (17) }\end{array}$ & $\begin{array}{l}\text { Davis } \\
\text { QHES }(37)^{3}\end{array}$ & $\begin{array}{l}\text { Winser } \\
\text { QHES (37) }\end{array}$ & $\begin{array}{l}\text { PHE NICE } \\
\text { (Yes } \\
\#)(38)^{4}\end{array}$ & $\begin{array}{l}\text { PHE NICE } \\
\text { verdict (38) }\end{array}$ & $\begin{array}{l}\text { Olij } \\
\text { CHEC (39) }\end{array}$ & \multirow[t]{2}{*}{$\begin{array}{l}\text { Stand } \\
\text { deviat } \\
\text { of } \% \\
\text { scores }\end{array}$} \\
\hline & & & $\begin{array}{l}\text { Max: } 10 \text { items } \\
\text { converted \% }\end{array}$ & $\begin{array}{l}\text { Max: } 10 \text { items } \\
\text { converted \% }\end{array}$ & Max: $100 \%$ & $\begin{array}{l}\text { Max: } \\
100 \%\end{array}$ & $\begin{array}{l}\text { Max: } 19 \\
\text { items } \\
\text { converted } \\
\%\end{array}$ & Categories $^{5}$ & Max: $100 \%$ & \\
\hline \multicolumn{11}{|c|}{ Single-vehicle evaluations (SVEs) (e.g., alongside randomized controlled trials) } \\
\hline 1 & $\begin{array}{l}\text { Campbell } \\
(2005)(40)\end{array}$ & $\begin{array}{l}\text { Davis; } \\
\text { PHE; Olij }\end{array}$ & 90.0 & & 100 & & 47.4 & $\begin{array}{l}\text { Partially / } \\
\text { Minor }\end{array}$ & 78.0 & 22.8 \\
\hline 2 & $\begin{array}{l}\text { Cockayne } \\
(2017)(41)\end{array}$ & Olij & & & & & & & 63.0 & \\
\hline 3 & $\begin{array}{l}\text { Davis } \\
(2011)(42)\end{array}$ & $\begin{array}{l}\text { DJ; Olij; } \\
\text { Winser }\end{array}$ & & 100 & & 99.0 & & & 89.0 & 6.1 \\
\hline 4 & $\begin{array}{l}\text { Davis } \\
\text { (2011b) (43) }\end{array}$ & Winser & & & & 87.0 & & & & \\
\hline 5 & $\begin{array}{l}\text { Farag } \\
(2015)(44)\end{array}$ & Olij & & & & & & & 92.0 & \\
\hline 6 & $\begin{array}{l}\text { Farag } \\
(2016)(45)\end{array}$ & PHE & & & & & 57.9 & $\begin{array}{l}\text { Partially / } \\
\text { Minor }\end{array}$ & & \\
\hline 7 & $\begin{array}{l}\text { Fletcher } \\
(2012)(46)\end{array}$ & PHE & & & & & 57.9 & $\begin{array}{l}\text { Directly / } \\
\text { Minor }\end{array}$ & & \\
\hline 8 & $\begin{array}{l}\text { Hendriks } \\
(2008)(47)\end{array}$ & $\begin{array}{l}\text { DJ; PHE; } \\
\text { Olij; Winser }\end{array}$ & & 100 & & 93.0 & 84.2 & $\begin{array}{l}\text { Partially / } \\
\text { Minor }\end{array}$ & 89.0 & 6.7 \\
\hline 9 & $\begin{array}{l}\text { Irvine } \\
(2010)(48)\end{array}$ & $\begin{array}{l}\text { DJ; PHE; } \\
\text { Olij; Winser }\end{array}$ & & 100 & & 79.0 & 52.6 & $\begin{array}{l}\text { Partially / } \\
\text { Minor }\end{array}$ & 92.0 & 20.7 \\
\hline 10 & $\begin{array}{l}\text { Isaranuwatchai } \\
(2017)(49)\end{array}$ & $\begin{array}{l}\text { Huter; } \\
\text { Winser }\end{array}$ & & 70.0 & & 91.0 & & & & 14.8 \\
\hline 11 & $\begin{array}{l}\text { Jenkyn } \\
(2012)(50)\end{array}$ & $\begin{array}{l}\text { DJ; PHE; } \\
\text { Olij }\end{array}$ & & 90.0 & & & 42.1 & $\begin{array}{l}\text { Partially / } \\
\text { Minor }\end{array}$ & 79.0 & 25.1 \\
\hline 12 & $\begin{array}{l}\text { Kenkre } \\
(2002)(51)\end{array}$ & DJ & & 50.0 & & & & & & \\
\hline 13 & Li (2015) (52) & PHE & & & & & 57.9 & $\begin{array}{l}\text { Partially / } \\
\text { Minor }\end{array}$ & & \\
\hline 14 & $\begin{array}{l}\text { Patil } \\
\text { (2016) (53) }\end{array}$ & PHE; Olij & & & & & 52.6 & $\begin{array}{l}\text { Partially / } \\
\text { Minor }\end{array}$ & 97.0 & 31.4 \\
\hline 15 & $\begin{array}{l}\text { Peeters } \\
(2011)(54)\end{array}$ & PHE; Olij & & & & & 73.7 & $\begin{array}{l}\text { Directly / } \\
\text { Minor }\end{array}$ & 92.0 & 13.0 \\
\hline 16 & $\begin{array}{l}\text { Polinder } \\
\text { (2016) (55) }\end{array}$ & PHE & & & & & 63.2 & $\begin{array}{l}\text { Directly / } \\
\text { Minor }\end{array}$ & & \\
\hline 17 & $\begin{array}{l}\text { Rizzo } \\
(1996)(56)\end{array}$ & $\begin{array}{l}\text { RCN; } \\
\text { Davis; Olij }\end{array}$ & 80.0 & & 75.0 & 61.0 & & & 62.0 & 9.5 \\
\hline 18 & $\begin{array}{l}\text { Robertson } \\
\text { (2001a) (57) }\end{array}$ & $\begin{array}{l}\text { RCN; } \\
\text { Davis; DJ; } \\
\text { Olij }\end{array}$ & 90.0 & 80.0 & 100 & 66.0 & & & 91.0 & 13.0 \\
\hline 19 & $\begin{array}{l}\text { Robertson } \\
\text { (2001b) (58) }\end{array}$ & $\begin{array}{l}\text { RCN; } \\
\text { Davis; DJ; } \\
\text { Olij }\end{array}$ & 90.0 & 90.0 & 92.0 & & & & 91.0 & 1.0 \\
\hline 20 & $\begin{array}{l}\text { Robertson } \\
\text { (2001c) (59) }\end{array}$ & $\begin{array}{l}\text { Davis; DJ; } \\
\text { Olij }\end{array}$ & 90.0 & 80.0 & 100 & 93.0 & & & 94.0 & 7.3 \\
\hline 21 & $\begin{array}{l}\text { Sach } \\
(2012)(60)\end{array}$ & PHE & & & & & 68.4 & $\begin{array}{l}\text { Directly / } \\
\text { Minor }\end{array}$ & & \\
\hline 22 & $\begin{array}{l}\text { Salkeld } \\
(2000)(61)\end{array}$ & $\begin{array}{l}\text { RCN; } \\
\text { Davis; DJ; } \\
\text { Olij }\end{array}$ & 60.0 & 80.0 & 73.0 & & & & 85.0 & 10.8 \\
\hline \multirow[t]{2}{*}{23} & $\begin{array}{l}\text { Timonen } \\
(2008)(62)\end{array}$ & Winser & & & & 39.0 & & & & \\
\hline & & Average: & 83.3 & 84.0 & 90.0 & 78.7 & 59.8 & & 85.3 & 11.5 \\
\hline
\end{tabular}




\begin{tabular}{|c|c|c|c|c|c|c|c|c|c|c|}
\hline 1 & $\begin{array}{l}\text { Albert } \\
\text { (2016) (63) }\end{array}$ & PHE & & & & & 47.4 & $\begin{array}{l}\text { Partially / } \\
\text { Potentially } \\
\text { serious }\end{array}$ & & \\
\hline 2 & $\begin{array}{l}\text { Beard } \\
(2006)(64)\end{array}$ & Davis; PHE & 70.0 & & 59.0 & & 52.6 & $\begin{array}{l}\text { Directly / } \\
\text { Minor }\end{array}$ & & 8.8 \\
\hline 3 & $\begin{array}{l}\text { Carande-Kulis } \\
(2015)(65)\end{array}$ & DJ; PHE & & 80.0 & & & 42.1 & $\begin{array}{l}\text { Partially / } \\
\text { Minor }\end{array}$ & & 26.8 \\
\hline 4 & $\begin{array}{l}\text { Church } \\
(2011)(66)\end{array}$ & $\begin{array}{l}\text { DJ; PHE; } \\
\text { Olij }\end{array}$ & & 70.0 & & & 78.9 & $\begin{array}{l}\text { Partially / } \\
\text { Minor }\end{array}$ & 83.0 & 6.7 \\
\hline 5 & $\begin{array}{l}\text { Church } \\
(2012)(67)\end{array}$ & $\begin{array}{l}\text { DJ; PHE; } \\
\text { Olij }\end{array}$ & & 50.0 & & & 68.4 & $\begin{array}{l}\text { Directly / } \\
\text { Minor }\end{array}$ & 83.0 & 16.5 \\
\hline 6 & $\begin{array}{l}\text { Day } \\
(2009)(68)\end{array}$ & DJ & & 70.0 & & & & & & \\
\hline 7 & $\begin{array}{l}\text { Farag } \\
(2015 b)(69)\end{array}$ & $\begin{array}{l}\text { DJ; PHE; } \\
\text { Olij }\end{array}$ & & 70.0 & & & 68.4 & $\begin{array}{l}\text { Partially / } \\
\text { Minor }\end{array}$ & 75.0 & 3.4 \\
\hline 8 & $\begin{array}{l}\text { Frick } \\
(2010)(70)\end{array}$ & $\begin{array}{l}\text { DJ; PHE; } \\
\text { Olij }\end{array}$ & & 60.0 & & & 68.4 & $\begin{array}{l}\text { Partially / } \\
\text { Minor }\end{array}$ & 68.0 & 4.7 \\
\hline 9 & $\begin{array}{l}\text { Hektoen } \\
(2009)(71)\end{array}$ & DJ; PHE & & 90.0 & & & 26.5 & $\begin{array}{l}\text { Partially / } \\
\text { Potentially } \\
\text { serious }\end{array}$ & & 45.0 \\
\hline 10 & $\begin{array}{l}\text { Johansson } \\
\text { (2008) (72) }\end{array}$ & DJ & & 90.0 & & & & & & \\
\hline 11 & Lee (2013) (73) & Olij & & & & & & & 90.0 & \\
\hline 12 & $\begin{array}{l}\text { Ling } \\
(2008)(74)\end{array}$ & DJ & & 30.0 & & & & & & \\
\hline 13 & $\begin{array}{l}\text { McLean } \\
(2015)(36)\end{array}$ & $\begin{array}{l}\text { PHE; Olij; } \\
\text { Huter; } \\
\text { Winser }\end{array}$ & & 90.0 & & 94.0 & 73.7 & $\begin{array}{l}\text { Directly / } \\
\text { Minor }\end{array}$ & 95.0 & 9.9 \\
\hline 14 & $\begin{array}{l}\text { Mori } \\
(2017)(75)\end{array}$ & Olij & & & & & & & 93.0 & \\
\hline 15 & $\begin{array}{l}\text { OMAS } \\
(2008)(76)\end{array}$ & DJ & & 80.0 & & & & & & \\
\hline 16 & $\begin{array}{l}\text { Pega } \\
(2016)(77)\end{array}$ & PHE; Olij & & & & & 84.2 & $\begin{array}{l}\text { Directly / } \\
\text { Minor }\end{array}$ & 83.0 & 0.9 \\
\hline 17 & $\begin{array}{l}\text { Poole } \\
\text { (2015) (78) }\end{array}$ & Olij & & & & & & & 75.0 & \\
\hline 18 & $\begin{array}{l}\text { Sach } \\
(2007)(79)^{7}\end{array}$ & Davis; Olij & 100 & & 92.0 & & & & 89.0 & 5.7 \\
\hline 19 & $\begin{array}{l}\text { Smith } \\
\text { (1998) (80) }\end{array}$ & $\begin{array}{l}\text { RCN; } \\
\text { Davis; Olij }\end{array}$ & 70.0 & & 47.0 & & & & 76.0 & 15.3 \\
\hline 20 & $\begin{array}{l}\text { van der Velde } \\
(2008)(81)\end{array}$ & PHE & & & & & 52.6 & $\begin{array}{l}\text { Directly / } \\
\text { Minor }\end{array}$ & & \\
\hline \multirow[t]{2}{*}{21} & Wu (2010) (82) & PHE & & & & & 52.6 & $\begin{array}{l}\text { Directly / } \\
\text { Minor }\end{array}$ & & \\
\hline & & Average: & 80.0 & 70.9 & 66.0 & 94.0 & 59.6 & & 82.7 & 12.5 \\
\hline
\end{tabular}

Abbreviation: CHEC: Consensus on Health Economic Criteria; DJ: Dubas-Jakobczyk; FoF: fear of falling; HAM: home assessment and modification; NICE: National Institute for Health and Care Excellence; OMAS: Ontario Medical Advisory Secretariat; PHE: Public Health England; PS: public sector; QHES: Quality o Health Economic Studies; RCN: Royal College of Nursing; RCT: randomized controlled trial.

Shading: Evaluations shaded in light grey received consistent quality ranking across multiple reviews that applied a quantitative quality checklist. Consistent ranking is defined as receiving quality scores that are all above or below the average score for each checklist by study design. Evaluations in dark grey receivı inconsistent ranking.

${ }^{1}$ References for systematic reviews: RCN (16); Davis (30); DJ (34); PHE (31); Olij (32); Huter (35); Winser (33)

${ }^{2}$ Contents of the checklists are given in Table $\mathrm{C}$ in Supplementary material.

${ }^{3}$ Davis review removed item 4 from the QHES checklist to produce a maximum score of 99 . But it also calculated the percentage which is reported here.

${ }^{4}$ This column reports the number of 'Yes' in a 19-item checklist given to a study by the PHE review. Potential options are: 'Yes'; 'No'; 'Partly'; 'Unclear'; 'N/A'. PH review added an extra item (item 8; see Table C in Supplementary material) to the original checklist.

${ }^{5}$ Categories for applicability: 'Directly'; 'Partially'; 'Not'. Categories for general limitations: 'Minor'; 'Potentially serious'; 'Very serious'. 
${ }^{6}$ Olij review added an extra item (item 5; see Table C in Supplementary material) to the original checklist.

7 This study was included as a decision model because it extrapolated the results of a trial over a lifetime horizon.

Thirteen out of 24 SVEs and 11 out of 21 models received scores from multiple reviews. The last column of Table 4 shows the standard deviation (SD) of scores per evaluation. The SD varied markedly between evaluations, ranging from 0.9 to 45.0 . The average checklist scores were also calculated for each review by study design. By comparing an individual evaluation's score against the average, its relative quality ranking (above or below average) within each review could be determined. Table 4 highlighted in light grey the evaluations which consistently scored above or below average in all reviews that identified them, and in dark grey those whose relative ranking changed across reviews. Only 7 of the 13 SVEs that received multiple scores received consistent ranking, while only 5 of 11 models did. There were hence potential differences in how reviews perceived the relative quality of their included evaluations based on the checklist scores (though the relative rankings would also depend on what evaluations are included). For example, Hektoen (2009) received the Drummond checklist score of $90.0 \%$ in the DJ review and was above the review average for models (70.9\%); but it received NICE checklist score of $26.3 \%$ in the PHE review which was markedly below the review average for models (59.6\%).

In addition to checklists, the DJ review narratively synthesised limitations of included studies around the following methodological themes: identifying and measuring costs and benefits; uncertainty over input variables; short time horizon; problems with sample (e.g., low participation); and problems with generalizability. The PHE review noted the main limitations of evaluations as perceived by the evaluation authors or reviewers but did not group them by themes. The Huter review narratively synthesised how evaluations handled the challenges of societal analysis, namely the incorporation of: (1) informal caregiving cost; (2) productivity cost; (3) unrelated cost in added life years; and (4) wider non-health effects. It was found that these challenges were handled in few evaluations; and when handled, were done using very heterogenous methods.

\section{Commissioning and research recommendations in systematic reviews}

Table 5 summarises the commissioning and research recommendations made by previous reviews. 
Table 5

Commissioning recommendations and research implications from previous systematic reviews of community-based falls prevention economic evaluations

\section{Review Commissioning recommendations}

RCN - No commissioning recommendation based on systematic review results

(16)

Davis

(30)

- "We conclude that single interventions (such as the Otago Exercise Programme) targeted at

high-risk groups can prevent the greatest number of falls at the lowest incremental costs." (p. 89)

\section{Research recommendations/implications}

- Development of a de novo decision model to inform NICE clinical guideline (16)

- "We recommend that future economic evaluations be guided in part by the checklists available for assessing economic evaluations." (p. 88)

- Development of guideline and checklist for falls prevention economic evaluations (21)

- "There is a need for... methods adjusted to particular character of health promotion and primary prevention strategies for older population." (p. 670)

- "The existing studies are characterized by huge differences in the methods applied as well as overall quality which limits the comparability and generalizability of the results." (p. 670)

PHE - Exercise interventions (p. 39-40): Tai Chi is consistently most cost-effective for mobile older (31) persons; group exercise for women aged 70+ cost-effective; Otago home exercise may be costsaving with high adherence; other home exercises are not cost-effective

- Multifactorial interventions (p. 40): paramedic-implemented protocol that followed NICE guideline was cost-saving and is generalizable to English setting; risk assessment without treatments not cost-effective

- HAM likely cost-effective but current evidence not generalizable to English setting (p. 40-41)

- Medication review likely cost-effective (p. 41)

Olij - "Home assessment programs were most cost-effective type of program [based on CUA] for (32) community-dwelling older adults." (p. 2197)

- "Multifactorial programs and other [e.g., exercise] programs were less favourable [based on CUA]." (p. 2202)

- "Older populations reported more favourable ICERs... [but] it is not possible to draw firm conclusions about age differences." (p. 2202)

- "Methodological differences between studies hampered direct comparison of the costeffectiveness of program types." (p. 2197)

\section{- Falls prevention economic model should carefully consider whether the intervention being modelled is appropriate for English} setting and given target population (p. 44).

- Development of a de novo decision model to inform commissioning of falls prevention by CCGs/local authorities (83) whether they target high-risk, low-risk, or mixed populations because the baseline
- "Future economic evaluations of falls prevention should be designed, conducted and reported in accordance with current guidelines for economic evaluations to increase comparability." (p. 2202)

- "Future studies should clearly report fall risk is an important determinant of cost-effectiveness." (p. 2202)

- Models should directly compare different falls intervention types (p. 2202)

- "Disregarding [the four features ${ }^{1}$ ] could implicitly lead to a discrimination of health promotion and disease prevention against older people." (p. 9)

- "More research is necessary on the different approaches for [the four features'] inclusion and on their respective effects on the outcomes." (p. 9)

- "We recommend future studies to test the benefits of adding scheduled walking to the falls prevention exercise protocol." (p. 76)

- "Research is needed to evaluate the efficacy of [group-based learning and home-based practice] programs, in particular in comparison to other programs that may require more resources." (p. 76)

- "Further research is needed... in developing and underdeveloped countries. (p. 69)

- "Future research is needed to systematically compare [exercise-only and multifactorial programs]." (p. 78)

Abbreviation: CCG: clinical commissioning group; CUA: cost-utility analysis; HAM: home assessment and modification; NICE: National Institute for Health and Care Excellence; PT: physiotherapist

${ }^{1}$ These are: (i) measurement and valuation of informal caregiving; (ii) accounting for productivity costs (including unpaid work); (iii) accounting for unrelated cost in added life years; and (iv) accounting for wider non-health effects of interventions.

Scarce cost-effectiveness evidence prevented the RCN review from making commissioning recommendations. The Davis review recommended singlecomponent Otago home exercise based on the most favourable cost-per-unit ratio. The DJ review reported three exercise interventions and a citywide multifactorial intervention that produced the lowest cost-per-unit ratios from 'Good' quality evaluations (those that received $90-100 \%$ Drummond checklist 
score). The PHE review based recommendations by intervention type on cost-per-unit ratios. The Olij review recommended HAM over exercise and multifactorial interventions for community-dwelling older persons based on incremental cost per QALY ratios under CUA. The Winser review listed the characteristics of an ideal exercise intervention based on those of interventions that yielded favourable cost-per-unit ratios. It also found that singlecomponent exercises produced more favourable ratios than exercises within multifactorial interventions but called for further direct comparisons.

For research implications, the RCN and PHE reviews determined that a de novo model is required to assist commissioning due to lack of current evidence. The Davis and Olij reviews recommended that future evaluations follow a validated guideline or checklist for economic evaluations. The Davis review later informed the development of the expert guideline/checklist for falls prevention economic evaluations (21). The Huter review stressed that future evaluations should incorporate the four methodological challenges associated with societal analyses (given above) to counteract the indirect bias of economic evaluations against older age groups (e.g., due to reduced scope of QALY gain). It should nevertheless be noted that inclusion of productivity costs would favour economically active/younger populations (see the results of Johansson (2008) (72) in Table 6 below where addition of net consumption changed the evaluation outcome from dominance to a relatively high ICER due to low productivity in old age).

\section{Critical appraisal of previous systematic review methodology}

According to the 11-item AMSTAR checklist, the systematic reviews were of comparable quality, with most scoring between 7 and 8 (see Table $\mathrm{D}$ in Supplementary material). Most prevalent issues were the non-provision of the list of excluded studies (item 5), the lack of assessment of publication bias (item 10), and whether the reviews adequately considered the scientific/methodological quality of included studies in formulating conclusions (item 8 ). Limitations acknowledged by the review authors included: limited search coverage (31-34); lack of quantitative meta-analysis (31, 33); non-assessment of publication bias $(31,32)$; and limited assessment of the quality of underlying clinical studies $(31,32)$.

Two further limitations of systematic reviews can be noted by this systematic overview:

1. The limited range of methodological features extracted from studies, particularly models; and

2. The limited range of evaluation outcomes extracted to inform commissioning.

The first limitation is made clear by comparing Tables 1 and 3. There was a marked difference between what data fields could or should have been extracted by systematic reviews according to expert guidelines and literature $(14,21-24)$ (Table 1 ) and those extracted (Table 3 ). Decision model features were the most neglected category. One particularly important (yet neglected) set of modelling features are methods for characterising the dynamic progression in falls risk and falls prevention intervention need. An individual's falls risk profile encompasses multiple interacting risk factors - including age, falls history, physical function (e.g., gait and balance) and cognitive function (15) - which are all highly dynamic; and changes to the falls risk profile would then entail changes to intervention need and eligibility. As far as time and resources permit, systematic reviews should account for how such features were modelled, including the data sources and parameters used and structural assumptions made. Insofar as models - and particularly population-level long-horizon models - provide the most relevant information to commissioners, the reviews' limited focus on the modelling features reduces their capacity to inform not only the commissioning decisions but also the conceptualisation of future falls prevention economic models.

The second limitation concerns the way in which reviews' commissioning recommendations were based chiefly on cost-per-unit ratios without considering aggregate outcomes. For example, the Davis review recommended the Otago home exercise for population aged 80+ based on a single SVE result that the intervention produced a net cost saving (57). Yet another evaluation in the review reported a similar cost saving from a citywide intersectoral intervention over a 5-year horizon (64). Even with comparable cost-per-unit ratios, consideration of aggregate impact would favour the citywide intervention. The cost-per-unit ratio also provides little information on the coverage of priority subgroups within the target population. For example, the Olij and Winser reviews recommended HAM and exercise, respectively, over multifactorial interventions based on comparisons of cost-per-unit ratios alone. Yet multifactorial interventions may achieve greater coverage of the most vulnerable patient groups (e.g., those contraindicated for exercise) and hence may be preferred by commissioners who aim to prioritise the care of such groups. Alternatively, HAM/exercise and multifactorial intervention may be commissioned as non-mutually exclusive options, with the more cost-effective option subsidising the lesser. The cost-per-unit ratios estimated in the absence of any capacity constraint should also be interpreted with caution since they would rise quickly once the intervention scale reaches the capacity limit.

\section{Commissioning recommendation by this systematic overview}

Assuming that decision-makers overseeing a health jurisdiction (e.g., at city, state or national level) would prefer general population, lifetime evidence to capture the full health and economic impacts of falls for the whole jurisdiction rather than specific patient groups $(21,29)$, Table 6 summarises the characteristics and results of five general population, lifetime models that were identified by the previous systematic reviews. Two principles are maintained in interpreting the model results: (I) attention is paid to methodological features that may influence the outcomes or the applicability of the outcomes to the decision-making setting (see category (D) in Table 1); and (II) recommendation is based on a wide range of reported outcomes, not cost-per-unit ratio alone (see category (E) in Table 1). 
Table 6

Characteristics and results of lifetime modelling studies identified by included systematic reviews

\begin{tabular}{|c|c|c|c|c|c|c|}
\hline Study & $\begin{array}{l}\text { Analysis; } \\
\text { Perspective }\end{array}$ & $\begin{array}{l}\text { Target } \\
\text { population }\end{array}$ & $\begin{array}{l}\text { Falls } \\
\text { epidemiology }\end{array}$ & Intervention features & Evaluation results ${ }^{1}$ & Methodological caveats \\
\hline $\begin{array}{l}\text { Church } \\
(2012) \\
(67)\end{array}$ & $\begin{array}{l}\text { CEA/CUA; } \\
\text { Public } \\
\text { healthcare }\end{array}$ & $\begin{array}{l}\text { CD adults } \\
\text { aged } 65+\end{array}$ & $\begin{array}{l}\text { Data source: } \\
\text { literature; } \\
\text { expert } \\
\text { opinion } \\
\text { Fall type: } \\
\text { non-MA fall; } \\
\text { MA fall; hip } \\
\text { fracture; fear } \\
\text { of falling; } \\
\text { fatal fall } \\
\text { Economic: } \\
\text { ED; inpatient; } \\
\text { rehab.; LTC }\end{array}$ & $\begin{array}{l}\text { Type: (i) General - Group } \\
\text { exercise; Home exercise; Tai } \\
\text { Chi; Multi-component int.; } \\
\text { Multifactorial int.; } \\
\text { Multifactorial risk } \\
\text { assessment; (ii) High-risk - } \\
\text { Group exercise; HAM; } \\
\text { Multifactorial int.; (iii) } \\
\text { Specific - Expedited } \\
\text { cataract surgery; Cardiac } \\
\text { pacing; Psychotropics } \\
\text { withdrawal } \\
\text { Comparator: UC; Cross } \\
\text { comparisons } \\
\text { Resource/cost: Per- } \\
\text { participant cost only } \\
\text { Implementation: 1-year } \\
\text { maintenance }\end{array}$ & $\begin{array}{l}\text { Ratios: (i) General - Tai Chi } \\
\text { ICER £27,734 per QALY vs. UC; } \\
\text { other interventions dominated; } \\
\text { (ii) High-risk - Group exercise } \\
\text { ICER £31,957 per QALY vs. UC; } \\
\text { HAM ICER £36,298 per QALY vs. } \\
\text { UC; Multifactorial int. } \\
\text { dominated; (iii) Specific - } \\
\text { Expedited cataract surgery } \\
\text { dominated UC and other } \\
\text { interventions. } \\
\text { Aggregate: reports incremental } \\
\text { cost, no. of falls avoided and } \\
\text { QALY gain per intervention, but } \\
\text { all interventions have same } \\
\text { reach }{ }^{3} \text { (including those targeting } \\
\text { high-risk and specific } \\
\text { subgroups), and hence cannot } \\
\text { compare aggregate impacts. } \\
\text { Parameter uncertainty: CEAC; } \\
\text { one-way sensitivity analyses on } \\
\text { ICER } \\
\text { Scenario analyses: No fear of } \\
\text { falling had substantial impact }\end{array}$ & $\begin{array}{l}\text { Unclear falls risk } \\
\text { progression }{ }^{4} \text {; Recurrent } \\
\text { falls not characterised } \\
\text { Unclear intervention }^{5} \\
\text { reach }^{3} \text {; Unclear how } \\
\text { high-risk subgroup } \\
\text { identified; Mismatch } \\
\text { between falls incidence } \\
\text { and efficacy metrics }{ }^{6} \text {; No } \\
\text { fixed int. cost; No } \\
\text { capacity constraints }\end{array}$ \\
\hline $\begin{array}{l}\text { Farag } \\
(2015) \\
(69)\end{array}$ & $\begin{array}{l}\text { CUA; Public } \\
\text { healthcare }\end{array}$ & $\begin{array}{l}\text { CD adults } \\
\text { aged } 65+ \\
\text { without } \\
\text { prior fall }\end{array}$ & $\begin{array}{l}\text { Data source: } \\
\text { literature } \\
\text { Fall type: } \\
\text { non-MA fall; } \\
\text { MA fall; fatal } \\
\text { fall } \\
\text { Economic: } \\
\text { ED; inpatient; } \\
\text { LTC }\end{array}$ & $\begin{array}{l}\text { Type: Non-specific falls } \\
\text { prevention int. with relative } \\
\text { risk of } 0.75 \text { and per- } \\
\text { participant cost of } £ 420 \\
\text { Comparator: UC } \\
\text { Resource/cost: Per- } \\
\text { participant cost only } \\
\text { Implementation: } 50 \% \text { uptake } \\
\text { in base case; maintenance }{ }^{2} \\
\text { not stated }\end{array}$ & $\begin{array}{l}\text { Ratios: ICER of } £ 17,320 \text { per } \\
\text { QALY vs. UC } \\
\text { Aggregate: incremental cost and } \\
\text { QALY gain outcomes per person } \\
\text { can be scaled up but unclear to } \\
\text { what extent. } \\
\text { Parameter uncertainty: CEAC; } \\
57 \% \text { probability of being cost- } \\
\text { effective at AUS } \$ 50,000 \\
\text { threshold; one-way sensitivity } \\
\text { analyses on ICER } \\
\text { Scenario analyses: e.g., } \\
\text { variation in uptake rate had little } \\
\text { impact on ICER }\end{array}$ & $\begin{array}{l}\text { Unclear falls risk } \\
\text { progression }{ }^{4} \text {; Recurrent } \\
\text { falls not characterised } \\
\text { No discounting; No fixed } \\
\text { int. cost; No capacity } \\
\text { constraints }\end{array}$ \\
\hline $\begin{array}{l}\text { Johansson } \\
\text { (2008) } \\
(72)\end{array}$ & $\begin{array}{l}\text { CUA; } \\
\text { Societal }\end{array}$ & $\begin{array}{l}\text { CD adults } \\
\text { aged } 65+ \\
(n=5,500)\end{array}$ & $\begin{array}{l}\text { Data source: } \\
\text { int. study } \\
\text { Fall type: hip } \\
\text { fracture; } \\
\text { excess } \\
\text { mortality } \\
\\
\text { Economic: } \\
\text { primary care; } \\
\text { inpatient; } \\
\text { outpatient; } \\
\text { pharma.; } \\
\text { LTC; informal } \\
\text { care; } \\
\text { productivity } \\
\text { loss; } \\
\text { comorbidity } \\
\text { net } \\
\text { consumption } \\
\text { cost }\end{array}$ & $\begin{array}{l}\text { Type: Multifactorial and } \\
\text { environmental int. }^{8} \\
\text { Comparator: UC } \\
\text { Resource/cost: Reports total } \\
\text { int. cost; Includes cost of } \\
\text { stakeholder involvement, } \\
\text { volunteer labour and time } \\
\text { opportunity cost }^{9} \\
\text { Implementation: not stated }\end{array}$ & $\begin{array}{l}\text { Ratio: intervention had higher } \\
\text { health gain and lower cost } \\
\text { (dominated) comparator } \\
\text { Aggregate: total int. cost of } \\
£ 640,918 ; \text { total costs savings of } \\
£ 647,970 ; \text { total QALY gain of } \\
35.16 \\
\text { Parameter uncertainty: Scatter } \\
\text { plot } \\
\text { Scenario analyses: Scenarios } \\
\text { that made intervention no longer } \\
\text { dominant - doubled fracture } \\
\text { risk; lower treatment cost of } \\
\text { fracture; inclusion of } \\
\text { comorbidity net consumption } \\
\text { cost }{ }^{7} \text {; higher discount rate; no } \\
\text { health/cost consequences of } \\
\text { fracture beyond 1st year; } 25 \% \\
\text { rise in int. cost }\end{array}$ & $\begin{array}{l}\text { Unclear falls risk } \\
\text { progression }{ }^{4} \text {; Quasi- } \\
\text { experimental study for } \\
\text { effectiveness evidence; } \\
\text { No tiered threshold for } \\
\text { evaluating societal } \\
\text { outcomes }^{10} \text {; Internal and } \\
\text { external validation } \\
\text { conducted }^{11}\end{array}$ \\
\hline
\end{tabular}




\begin{tabular}{|c|c|c|c|c|c|c|}
\hline Study & $\begin{array}{l}\text { Analysis; } \\
\text { Perspective }\end{array}$ & $\begin{array}{l}\text { Target } \\
\text { population }\end{array}$ & $\begin{array}{l}\text { Falls } \\
\text { epidemiology }\end{array}$ & Intervention features & Evaluation results $^{1}$ & Methodological caveats \\
\hline $\begin{array}{l}\text { OMAS } \\
(2008) \\
(76)\end{array}$ & $\begin{array}{l}\text { CEA/ROI; } \\
\text { Public } \\
\text { healthcare }\end{array}$ & $\begin{array}{l}\text { CD adults } \\
\text { aged } 65+\end{array}$ & $\begin{array}{l}\text { Data source: } \\
\text { routine data } \\
\text { analysis } \\
\text { Fall type: MA } \\
\text { fall; excess } \\
\text { mortality } \\
\text { Economic: } \\
\text { ED; inpatient; } \\
\text { rehab.; LTC }\end{array}$ & $\begin{array}{l}\text { Type: Exercise; HAM; Vitamin } \\
\text { D \& calcium; Psychotropics } \\
\text { withdrawal; Gait stabilizing } \\
\text { device; Eligibility for each } \\
\text { intervention defined by } \\
\text { relevant falls risk factor }{ }^{12} \\
\text { Comparator: UC } \\
\text { Resource/cost: Per- } \\
\text { participant cost only } \\
\text { Implementation: Unique } \\
\text { uptake and adherence rates } \\
\text { for each intervention; } \\
\text { Permanent maintenance }{ }^{2} \text { for } \\
\text { 1st year adherers }\end{array}$ & $\begin{array}{l}\text { Ratio: All interventions } \\
\text { dominated UC under CEA for } \\
\text { men and women } \\
\text { Aggregate: Reports net cost } \\
\text { saving per person which can be } \\
\text { scaled up to total for each } \\
\text { intervention subgroup at } \\
\text { regional level } \\
\text { Parameter uncertainty: No } \\
\text { analysis } \\
\text { Scenario analyses: No analysis }\end{array}$ & $\begin{array}{l}\text { Unclear falls risk } \\
\text { progression }{ }^{4} \text {; Recurrent } \\
\text { falls not characterised } \\
\text { Mismatch between } \\
\text { intervention need and } \\
\text { falls risk }^{12} \text {; Parameter } \\
\text { uncertainty not assessed }^{5}\end{array}$ \\
\hline $\begin{array}{l}\text { Pega } \\
(2016) \\
(77)\end{array}$ & $\begin{array}{l}\text { CUA; Public } \\
\text { healthcare }\end{array}$ & $\begin{array}{l}\text { CD adults } \\
\text { aged } 65+\end{array}$ & $\begin{array}{l}\text { Data source: } \\
\text { routine data } \\
\text { analysis } \\
\text { Fall type: } \\
\text { indoor MA } \\
\text { fall; fatal fall } \\
\text { Economic: } \\
\text { primary care; } \\
\text { pharma.; } \\
\text { rehab.; } \\
\text { inpatient; } \\
\text { comorbidity } \\
\text { healthcare } \\
\text { cost }^{13}\end{array}$ & $\begin{array}{l}\text { Type: HAM } \\
\text { Comparator: UC } \\
\text { Resource/cost: Per- } \\
\text { participant cost only } \\
\text { Implementation: One-off } \\
\text { HAM yields lifetime efficacy } \\
\text { (10 years in scenario) }\end{array}$ & $\begin{array}{l}\text { Ratio: HAM had ICER of } £ 5,123 \\
\text { per QALY vs. UC in base case } \\
\text { Aggregate: For base case, total } \\
\text { int. cost was } £ 82.5 \text { million, total } \\
\text { net cost vs. UC } £ 62.6 \text { million } \\
\text { and total QALY gain } 34,000 \text {. } \\
\text { Parameter uncertainty: } 95 \% \\
\text { uncertainty interval for ICER } \\
\text { between below zero to } £ 11,385 \\
\text { per QALY; one-way sensitivity } \\
\text { analyses } \\
\text { Scenario analyses: For } \\
\text { secondary prevention } \\
\text { scenario }{ }^{14} \text {, ICER was } £ 1,139 \text { per } \\
\text { QALY, total int. cost } £ 10.2 \\
\text { million, total net cost vs. UC, } \\
£ 3.5 \text { million, and total QALY gain } \\
20,100 \text {. } \\
\text { Subgroup analyses showed } \\
\text { higher ICERs for Maori and men. } \\
\text { Equity analyses showed that the } \\
\text { higher ICERs can be mainly } \\
\text { attributed to shorter life } \\
\text { expectancies of Maori and } \\
\text { men } 15 \text {. }\end{array}$ & $\begin{array}{l}\text { Unclear falls risk } \\
\text { progression }{ }^{4} \text {; Recurrent } \\
\text { falls not characterised } \\
\text { Routine data lacks } \\
\text { individual identifier } \\
\text { back; No } \\
\text { backound transition in } \\
\text { health utilities; No fixed } \\
\text { int. cost; No capacity } \\
\text { constraints; No scenario } \\
\text { estimating efficiency } \\
\text { cost }^{15}\end{array}$ \\
\hline
\end{tabular}




$\begin{array}{lllllll}\text { Study } & \text { Analysis; } & \text { Target } & \text { Falls } & \text { Intervention features } & \text { Evaluation results } & \end{array}$

Abbreviation: CEA: cost-effectiveness analysis; CD: community-dwelling; CUA: cost-utility analysis; ED: emergency department; HAM: home assessment and modification; ICER: incremental cost-effectiveness ratio; int.: intervention; LTC: Iong-term care admission; MA fall: fall requiring medical attention; OMAS: Ontario Medical Advisory Secretariat; pharma.: pharmaceuticals; QALY: quality-adjusted life year; rehab.: rehabilitation; ROI: return on investment; UC: usual care

${ }^{1}$ All monetary units are converted to $£$ in year 2021 using the average consumer price index (CPI) between the original year of reported currency to 2019 (most recent year for CPI data) in the country of study and purchasing power parity (PPP) rate between the original currency and $£$ in year 2020 (most recent PPP data)

${ }^{2}$ Maintenance refers to the duration of eligible persons receiving the intervention. Intervention effectiveness is a function of efficacy durability and maintenance period.

${ }^{3}$ Intervention reach refers to the number/proportion of persons receiving the intervention. It is a function of intervention's normative reach defined by its eligibility criteria and targeting strategy and its implementation reach determined by the level of implementation (e.g., uptake and adherence) within the eligible population.

${ }^{4}$ Specifically, the study does not mention how falls risk progressed with age in the absence of falls incidence (which has a separate model state). Markov model should incorporate tunnel states to allow for secular risk progression, but this is not stated or graphically illustrated.

${ }^{5}$ Markov models with 1-year cycles should assign the number of falls to individual fallers who experience at least one fall in a given 1-year cycle or include a separate model state for being a recurrent faller. Not incorporating recurrent falls would underestimate the health burden of falls.

${ }^{6}$ Falls incidence metrics are numbers of falls (falls rate) and fallers (falls risk). Matching efficacy metrics are falls rate ratio (RaR) and relative risk (RR) of falling.

${ }^{7}$ The study incorporated cost of added life-years which was estimated as the consumption minus production level (i.e., net consumption) that varied by age group. The outcome changed from dominance to ICER of $£ 16,980$ per QALY.

${ }^{8}$ Multifactorial intervention in this study included tailored education, group balance exercises, Tai Chi, other physical activities and HAM. Environmental intervention included neighbourhood hazard removal and housing reconstruction.

${ }^{9}$ Not all intervention costs were reimbursed by the public sector, meaning that there were societal intervention costs.

10 Societal costs incur different opportunity cost to public sector costs. The cost-effectiveness threshold should be tiered or weighted to capture the differing opportunity costs across sectors.

11 The study reported that the model slightly underestimated the hip fracture incidence relative to literature and that the model was checked for technical errors.

12 The study estimated the proportion of target population who would be eligible for each of the interventions according to the prevalence of falls risk factors that defined eligibility: exercise for mobile older persons without disability (65.8\%); HAM for frail older persons with disability (16.9\%); vitamin D for women with fracture risk factors (52.9\% of female); psychotropics withdrawal for psychotropic users (11.8\%); and gait stabilizers for mobile seniors without disability (65.8\%). However, the falls risk in the model was determined only by age, sex and MA falls history. Hence, different intervention subgroups had similar falls risk despite contrasting risk factor profiles.

${ }^{13}$ The study incorporated healthcare cost of added life-years and cost of dying (healthcare cost in last 6 months) which varied by age group and sex.

14 This scenario involved HAM targeted at subgroup with history of MA fall. This subgroup comprised $10 \%$ of target population.

15 The study evaluated counterfactual scenarios where Maori/men had equal life expectancy as non-Maori/women and found that subgroup ICERs became similar. This, however, does not estimate the efficiency cost incurred if Maori/men are prioritised for intervention under the factual circumstance of lower life expectancy.

${ }^{16}$ Without individual identifiers, multiple falls experienced by the same person are counted as multiple fallers.

Concerning principle (I), two salient features emerge from Table 6. First, as shown in the falls epidemiology column, there is significant between-study variation in the fall-related health and economic consequences incorporated and in the data sources used to characterise falls risk. Hence, the decision-maker preference over the range of fall-related health and economic consequences would influence the results' applicability. Secondly, each evaluation has several methodological caveats (see last column) that may affect the credibility of model results. For example, all five studies developed Markov cohort models but mentioned no tunnel states to account for the secular age-related increase in falls risk, which would bias the result against those who are younger at baseline (and against early prevention). Only Johansson (2008) assessed the model's external validity. The decision-maker should consider these methodological shortcomings when using the model evidence.

Four models that conducted CUA produced cost-per-unit ratios for at least one intervention relative to usual care that can be deemed cost-effective under the cost-effectiveness threshold of $£ 30,000$ per QALY gain (i.e., the NICE health technology assessment threshold (84)). In the order of increasing ICER values, the results were:

- Combined multifactorial and environmental intervention in Johansson (2008) with QALY gain and lower cost relative to usual care (72);

- HAM in Pega (2016) with ICER of $£ 5,123$ per QALY if implemented for the whole population and $£ 1,139$ if targeted at those with history of falls requiring medical attention (MA falls) (77);

- A non-specific intervention in Farag (2015) of $£ 420$ per-participant cost and $25 \%$ reduction in falls risk with ICER of $£ 17,320$ per QALY (69);

- Tai Chi in Church (2012) with ICER of $£ 27,734$ per QALY (67). 
Given the favourable ratios, a key decisional factor under principle (II) is the population reach of each intervention that determines its aggregate impact, as well as any budget and capacity constraints of the decision-maker. For example, it may be the case that Tai Chi enjoys a substantially greater uptake rate than HAM in the decision-making setting (perhaps due to high prevalence of rented accommodations which makes home modification difficult (77)). In this case, Tai Chi would generate greater aggregate gain (measured by incremental net monetary benefit that incorporates QALY gain and net costs) than HAM despite its higher ICER. But if there are significant budget or capacity constraints such that the wide Tai Chi uptake cannot be realised, then HAM would be preferred since it delivers more health gain per monetary unit of investment. A similar comparison should be made between universal provision of HAM and its targeted provision in Pega (2016). The targeted approach generates lower ICER but generates lower total QALY gain than universal provision: 20,100 QALYs at $£ 3.5$ million total net cost vs. 34,000 QALYs at $£ 62.6$ million total net cost. The additional 13,900 QALYs from universal provision is of greater value than the $£ 59.1$ million additional net cost if the cost-effectiveness threshold is greater than $£ 4,252$ per QALY. Thus, the targeted approach should be pursued only if there are budget/capacity constraints (or an equity objective; see below) that preclude the universal provision.

The combined multifactorial and environmental intervention in Johansson (2008) potentially has the greatest reach since it addresses community-wide environmental risk factors (independently of demand by older people) as well as individually tailored treatments including Tai Chi and HAM. However, the model is based on evidence from a quasi-experimental study in a small community of 5,500 older people, and there is no supplementary evidence that it can be successfully implemented in other communities. Hence, the decision-maker should first consult local stakeholders to determine whether the intervention in Johansson (2008) can be scaled up within the budget and capacity constraints. Whether older people's productivity is considered in the evaluation is another decisional factor since the outcome changes from dominance to ICER of $£ 16,890$ per QALY if net consumption cost in added life-years is included.

OMAS (2008) was the only model to conduct CEA for five single-component interventions relative to usual care: exercise, HAM, vitamin D \& calcium, psychotropics withdrawal, and gait stabilising device (76). All interventions reduced the number of MA falls and the net healthcare cost, thus dominating usual care. Gait stabilising device produced the highest reduction in MA falls and net cost and had the greatest population reach (65.8\%) and hence should be the preferred option. However, there were two main methodological caveats. First, no assessment of parameter uncertainty was conducted despite the paucity of evidence for several model parameters (e.g., only one trial was available for efficacy of gait stabilising device). Secondly, the population reaches of interventions were not based on the characteristics of the simulated model population but imposed exogenously. For example, gait stabilising device was eligible only for mobile seniors without disability, and according to an external survey, this group comprised $65.8 \%$ of the general geriatric population. The study then simply assumed that $65.8 \%$ of health gains and costs accrue to this intervention subgroup. But the simulated model population were defined by age, sex and MA falls history, not mobility or disability, and hence the true reach of gait stabilising device is unknown. These caveats reduced the credibility of the reported results.

Another key decisional factor under principle (II) is equity consideration beyond cost-effectiveness. Here, only Pega (2016) disaggregated the evaluation results into social subgroups: female vs. male; and non-Maori majority vs. Maori ethnic minority in New Zealand. Male and Maori subgroups had higher ICERs than their respective counterparts, and gained less QALYs per person (e.g., 0.046 for Maori vs. 0.060 for non-Maori). Hence, universal HAM provision worsens the health inequity between Maori and non-Maori (the decision-maker may similarly see the health inequality between men and women as unfair). Though the specific ethnic divide is unique to New Zealand, the decision-maker may choose to generalise this case to predict the distribution of HAM impact across locally relevant gradient in social marginalisation. Having done so, commissioning can consider HAM strategies that do not exacerbate the existing health inequity e.g., targeting the socially marginalised group - even at the expense of reduced cost-effectiveness. Similar considerations are warranted for other costeffective interventions, but there are insufficient subgroup results from other models to enable this.

Pega (2016) also provides an insight into the underlying cause of inequitable subgroup impacts. A scenario analysis is conducted wherein the Maori subgroup is assigned the longer life expectancy of the non-Maori subgroup, and it is found that Maori's QALY gain becomes higher than that of non-Maori (0.071 vs. 0.060) and the ICERs become similar. Hence, the inequitable impact can be attributed mainly to the life expectancy differential between ethnic subgroups though other potential causes of inequitable impact (e.g., lower intervention uptake or efficacy among the Maori) cannot be investigated due to homogenous parameter assumptions across ethnic subgroups. This suggests that falls prevention commissioning should be complemented by upstream interventions at earlier life stages to correct the life expectancy differential that emerges at age 65.

Overall, the commissioning recommendations of this systematic overview are as follows:

(1) There is some evidence that combined multifactorial and environmental intervention, HAM and Tai Chi are cost-effective over the lifetime for general geriatric populations aged $65+$.

(2) The decision-maker should investigate the feasible reaches of the above interventions in the local setting within the budget and capacity constraints. Commissioning of additional implementation support (e.g., peer motivators) can also be considered.

(3) There is some evidence that national provision of HAM exacerbates the existing health inequity across social subgroups, and this may generalise to the other two interventions. The decision-maker could consider targeting the intervention at socially marginalised groups or a universal provision supplemented by additional implementation support for the marginalised groups. Upstream interventions at early life stages can also supplement falls prevention.

(4) There are methodological caveats that may significantly influence the model outcomes. The decision-maker could consider commissioning the development of a de novo general population, lifetime model that addresses the main methodological challenges, such as the dynamic complexity in falls risk profile and the psychological and sociological factors that influence the intervention reach and hence its aggregate impact.

\section{Discussion}

This systematic overview identified 7 systematic reviews containing 44 falls prevention intervention economic evaluations for older people living in community. The number of data fields extracted from studies differed markedly across reviews, ranging from 8 to 33 . Four checklists were applied by reviews, 
while narrative quality assessment was conducted at varying levels of detail and topic range. Commissioning recommendations were based primarily on costper-unit ratios. Research recommendations ranged from a call for greater adherence to pre-established guidelines for economic evaluations to development of de novo decision models. The systematic overview made its own commissioning recommendations and critically appraised the methods of previous reviews, particularly regarding the extraction of methodological features and the synthesis of evaluation outcomes.

Application of the AMSTAR checklist to assess the reporting and methodological quality of systematic reviews yielded limited results: the 7 reviews received comparable AMSTAR scores despite the marked variation in the number of data fields extracted. A key issue is that AMSTAR, as well as its alternatives, AMSTAR2 (85) and ROBIS (86), recommended by the Cochrane guideline (19), are not designed specifically for assessing the quality of systematic reviews of economic evaluations, let alone of falls prevention economic evaluations. This systematic overview hence used expert guidelines on falls prevention economic evaluation and broader methodological literature on public health economic evaluation and modelling $(14,21-24)$ to formulate an independent set of criteria for determining the quality of systematic reviews. A similar approach was taken by a previous overview of systematic reviews of community pharmacy economic evaluations which formulated its quality assessment criteria (as well as applying AMSTAR2) based on a wide range of academic and policy literature on economic evaluation of public health interventions and trial-based economic evaluations (87). Future systematic overviews would likewise benefit from tailoring their quality assessment criteria to the disease area and study design of interest.

A noticeable finding of this overview was that previous systematic reviews of falls prevention economic evaluations neglected the extraction and analysis of decision model features. As mentioned, this greatly compromises the ability of systematic reviews to inform decision-making at the population level over a time horizon long enough to capture all relevant costs and consequences of a preventive intervention $(29,84)$. According to the systematic methodological review already referenced in Table 1, the key methodological challenges within public health economic model development include: (I) incorporating wider costs and effects; (II) considering dynamic complexity (e.g., long-term progression of falls risk factors); (III) incorporating psychological and sociological factors (e.g., those affecting intervention uptake/adherence); and (IV) considering social determinants of health and conducting equity analyses (24). The Huter review covered only (I), while the PHE review only (IV). Future systematic reviews of public health economic models should endeavour to cover as many of these aspects as possible. This would help judge the structural validity and credibility of included models before they inform commissioning decisions and/or conceptualisation of de novo falls prevention economic models. It would also inform additional commissioning strategies that could supplement falls prevention, such as upstream interventions to address the underlying social disadvantages resulting in inequitable impact of falls prevention (88), and implementation strategies to increase falls prevention uptake $(27,89-92)$.

A possible contributory factor to the neglect of decision model features is the nature of checklists used by previous systematic reviews to assess the reporting and methodological quality of their identified economic evaluations. All four checklists used by the reviews were designed for all disease areas and for all study designs. Though reviewers are not confined to extracting only the checklist items, the use of a generic, all-design checklist would likely reduce the effort spent in identifying how evaluations captured the disease- and modelling-specific features. Thus, using the fall-specific (but all-design) checklist designed by falls prevention experts (21) may improve the attention paid to features of falls epidemiology and falls prevention intervention by future systematic reviews, while using the model-specific (but generic) HTA checklist (23) may similarly improve the attention on modelling features. However, any quantitative checklist is likely too limited to serve as the main methodological assessment tool. Specifically, its use of binary/ordinal item scores, followed by aggregation to a single index, conceals the highly idiosyncratic nature of methodological issues and the way and extent to which they affect the evaluation outcomes (30). Hence, checklist application is necessary but insufficient to analyse the methodological quality of economic evaluations and must be complemented by a narrative synthesis of methodological features. This dual approach was adopted by few previous systematic reviews in this overview and hence remains a research priority for future systematic reviews.

Sole reliance on cost-per-unit ratios would generate incomplete and biased commissioning recommendations. As noted above, single-component HAM or exercise may generate very favourable cost-per-unit ratios and yet perform poorly in terms of aggregate impact and/or coverage of priority groups relative to a multifactorial intervention. This observation contributes to an ongoing debate on whether less resource-intensive exercise should be preferred over (the widely recommended) multifactorial interventions $(93,94)$. The debate is primarily centred around efficacy estimates and cost-per-unit ratios, but the final verdict cannot and should not be reached without considering the aggregate impact $(68,95)$ and decisional priorities beyond cost-effectiveness $(96)$. Consideration of aggregate outcomes is also important for informing targeting strategies (under budget/capacity constraints) and assessing the returns on intervention scaleup (27). Systematic reviews should therefore endeavour to extract a wide range of economic evaluation outcomes, though the feasible range would largely depend on the methodological and reporting practices of underlying evaluations.

\section{Strengths and limitations of this systematic overview}

This systematic overview is the first of its kind in the falls prevention economic evaluation context. It covered 12 academic databases and grey literature between 2003 and 2020 and followed the Cochrane guideline (19). It offered commissioning recommendations based on general population, lifetime models after considering their methodological caveats and outcomes beyond cost-per-unit ratios. It also critically appraised the methodological quality of previous systematic reviews, and this would help improve the quality of future systematic reviews' data extraction, quality assessment and formulation of commissioning recommendations. This would in turn aid the conceptualisation and implementation of future falls prevention economic evaluations, particularly those employing decision models.

The overview nevertheless has limitations, including non-coverage of the period before 2003, non-inclusion of systematic reviews of falls prevention RCTs that contained a minority of studies that were economic evaluations (10-12), and non-inclusion of reviews that targeted specific patient groups such as those with neurological disorders (97). The commissioning recommendations were made under certain assumptions on decision-maker preference - i.e., prioritization of general population, lifetime modelling evidence - and neglected evidence from SVEs and short-horizon models.

Page 19/25 


\section{Conclusion}

The systematic overview found significant variation and limitations in the methodological quality of existing systematic reviews of falls prevention economic evaluations which could bias commissioning decisions and hinder the design of future evaluations. Systematic reviews should: be as comprehensive as possible in the extraction and narrative synthesis of evaluation features associated with falls epidemiology, falls prevention intervention and decision modelling; they should also base the commissioning recommendations on the full range of reported outcomes and equity objectives to avoid biased and incomplete information being provided to decision-makers.

\section{Abbreviations}

AMSTAR: A MeaSurement Tool to Assess systematic Reviews

CEA: cost-effectiveness analysis

CG: clinical guideline

CHEERS: Consolidated Health Economic Evaluation Reporting Standards

CUA: cost-utility analysis

HAM: home assessment and modification

HTA: health technology assessment

ICER: incremental cost-effectiveness ratio

MA fall: fall requiring medical attention

PRISMA: Preferred Reporting Items for Systematic Reviews and Meta-Analyses

QALY: quality adjusted life year

NICE: National Institute for Health and Clinical Excellence

PHE: Public Health England

RCN: Royal College of Nursing

RCT: randomised controlled trial

ROBIS: Risk Of Bias In Systematic reviews tool

SVE: single-vehicle evaluation

\section{Declarations}

Ethics approval: None required.

Consent for publication: None required.

Availability of data and materials: The datasets used and analysed during the current study are available from the corresponding author on reasonable request.

Competing interests: The authors declare that they have no competing interests.

Funding: Mr Joseph Kwon was supported by the Wellcome Trust [108903/B/15/Z]

Authors' contributions: JK and YJ conducted the systematic search for study identification and data extraction. JK write the first manuscript draft. JK, HS, TY and MF contributed to the writing of subsequent drafts until manuscript submission. All authors read and approved the final manuscript.

Acknowledgements: We would like to thank Dr Andrew Booth at the School of Health and Related Research for kindly reviewing the manuscript and offering detailed feedback.

\section{References}

1. World Health Organization. WHO Global Report on Falls Prevention at Older Age. Geneva: WHO. 2007.

2. Gottschalk S, Konig H-H, Schwenk M, Jansen C-P, Nerz C, Becker C, et al. Mediating factors on the association between fear of falling and health-related quality of life in community-dwelling German older people: a cross-sectional study. BMC geriatrics. 2020;20(1):401. 
3. Kim JH. Experiences of falling and depression: Results from the Korean Longitudinal Study of Ageing. Journal of Affective Disorders. 2021;281:174-82.

4. Sekaran NK, Choi H, Hayward RA, Langa KM. Fall-associated difficulty with activities of daily living in functionally independent individuals aged 65 to 69 in the United States: a cohort study. J Am Geriatr Soc. 2013;61(1):96-100.

5. Drevet S, Bornu BC, Boudissa M, Bioteau C, Mazière S, Merloz P, et al. One-year mortality after a hip fracture: prospective study of a cohort of patients aged over 75 years old. Geriatrie et psychologie neuropsychiatrie du vieillissement. 2019;17(4):369-76.

6. Tian Y, Thompson J, Buck D, Sonola L. Exploring the system-wide costs of falls in older people in Torbay: King's Fund; 2013.

7. Davis J, Robertson M, Ashe M, Liu-Ambrose T, Khan K, Marra C. International comparison of cost of falls in older adults living in the community: a systematic review. Osteoporosis international. 2010;21(8):1295-306.

8. Kuzuya M, Masuda Y, Hirakawa Y, Iwata M, Enoki H, Hasegawa J, et al. Falls of the elderly are associated with burden of caregivers in the community. International Journal of Geriatric Psychiatry: A journal of the psychiatry of late life and allied sciences. 2006;21(8):740-5.

9. Cook J. The socio-economic contribution of older people in the UK. Working with Older People. 2011.

10. Gillespie LD, Robertson MC, Gillespie WJ, Sherrington C, Gates S, Clemson LM, et al. Interventions for preventing falls in older people living in the community. Cochrane database of systematic reviews. 2012(9).

11. Hopewell S, Adedire O, Copsey BJ, Boniface GJ, Sherrington C, Clemson L, et al. Multifactorial and multiple component interventions for preventing falls in older people living in the community. Cochrane database of systematic reviews. 2018(7).

12. Sherrington C, Fairhall NJ, Wallbank GK, Tiedemann A, Michaleff ZA, Howard K, et al. Exercise for preventing falls in older people living in the community. Cochrane database of systematic reviews. 2019(1).

13. Moher D, Liberati A, Tetzlaff J, Altman DG, Group P. Preferred reporting items for systematic reviews and meta-analyses: the PRISMA statement. PLoS medicine. 2009;6(7):e1000097.

14. Squires H, Chilcott J, Akehurst R, Burr J, Kelly MP. A framework for developing the structure of public health economic models. Value in Health. 2016;19(5):588-601.

15. National Institute for Health and Care Excellence. Falls in older people: assessing risk and prevention. National Institute for Health and Care Excellence. 2013;Clinical Guideline 161(nice.org.uk/guidance/cg161).

16. Royal College of Nursing. Clinical practice guideline for the assessment and prevention of falls in older people. Clinical Practice Guidelines. 2005;London: Royal College of Nursing.

17. Drummond MF, Sculpher MJ, Claxton K, Stoddart GL, Torrance GW. Methods for the economic evaluation of health care programmes: Oxford university press; 2015.

18. Husereau D, Drummond M, Petrou S, Carswell C, Moher D, Greenberg D, et al. Consolidated health economic evaluation reporting standards (CHEERS) statement. Cost Effectiveness and Resource Allocation. 2013;11(1):6.

19. Pollock M, Fernandes RM, Becker LA, Pieper D, Hartling L. Chapter V: Overviews of Reviews. In: In: Higgins JPT TJ, Chandler J, Cumpston M, Li T, Page MJ, Welch VA (editors), editor. Cochrane Handbook for Systematic Reviews of Interventions version 62 (updated February 2021$) 2018$.

20. Shea BJ, Grimshaw JM, Wells GA, Boers M, Andersson N, Hamel C, et al. Development of AMSTAR: a measurement tool to assess the methodological quality of systematic reviews. BMC medical research methodology. 2007;7(1):1-7.

21. Davis J, Robertson MC, Comans T, Scuffham P. Guidelines for conducting and reporting economic evaluation of fall prevention strategies. Osteoporosis international. 2011;22(9):2449-59.

22. Huter K, Kocot E, Kissimova-Skarbek K, Dubas-Jakóbczyk K, Rothgang H. Economic evaluation of health promotion for older people-methodological problems and challenges. BMC health services research. 2016;16(5):328.

23. Philips Z, Ginnelly L, Sculpher M, Claxton K, Golder S, Riemsma R, et al. Review of guidelines for good practice in decision-analytic modelling in health technology assessment. 2004.

24. Squires H, Chilcott J, Akehurst R, Burr J, Kelly MP. A systematic literature review of the key challenges for developing the structure of public health economic models. International journal of public health. 2016;61(3):289-98.

25. Lamb SE, Jørstad-Stein EC, Hauer K, Becker C, Europe PoFN, Group OC. Development of a common outcome data set for fall injury prevention trials: the Prevention of Falls Network Europe consensus. Journal of the American Geriatrics Society. 2005;53(9):1618-22.

26. Briggs A, Sculpher M, Claxton K. Decision modelling for health economic evaluation: Oup Oxford; 2006.

27. Fenwick E, Claxton K, Sculpher M. The value of implementation and the value of information: combined and uneven development. Medical Decision Making. 2008;28(1):21-32.

28. Cookson R, Drummond $\mathrm{M}$, Weatherly $\mathrm{H}$. Explicit incorporation of equity considerations into economic evaluation of public health interventions. Health Economics, Policy and Law. 2009;4(2):231-45.

29. Brennan A, Akehurst R. Modelling in health economic evaluation. Pharmacoeconomics. 2000;17(5):445-59.

30. Davis JC, Robertson MC, Ashe MC, Liu-Ambrose T, Khan KM, Marra CA. Does a home-based strength and balance programme in people aged $>$ or $=80$ years provide the best value for money to prevent falls? A systematic review of economic evaluations of falls prevention interventions. Br $\mathrm{J}$ Sports Med. 2010;44(2):80-9.

31. Public Health England. A structured literature review to identify cost-effective interventions to prevent falls in older people living in the community. Public Health England. 2018. 
32. Olij BF, Ophuis RH, Polinder S, Van Beeck EF, Burdorf A, Panneman MJ, et al. Economic evaluations of falls prevention programs for older adults: a systematic review. Journal of the American Geriatrics Society. 2018;66(11):2197-204.

33. Winser SJ, Chan HTF, Ho L, Chung LS, Ching LT, Felix TKL, et al. Dosage for cost-effective exercise-based falls prevention programs for older people: a systematic review of economic evaluations. Annals of physical and rehabilitation medicine. 2020;63(1):69-80.

34. Dubas-Jakóbczyk K, Kocot E, Kissimova-Skarbek K, Huter K, Rothgang H. Economic evaluation of health promotion and primary prevention actions for older people-a systematic review. The European Journal of Public Health. 2017;27(4):670-9.

35. Huter K, Dubas-Jakóbczyk K, Kocot E, Kissimova-Skarbek K, Rothgang H. Economic evaluation of health promotion interventions for older people: do applied economic studies meet the methodological challenges? Cost Effectiveness and Resource Allocation. 2018;16(1):14.

36. McLean K, Day L, Dalton A. Economic evaluation of a group-based exercise program for falls prevention among the older community-dwelling population. BMC Geriatr. 2015;15:33.

37. Ofman JJ, Sullivan SD, Neumann PJ, Chiou C-F, Henning JM, Wade SW, et al. Examining the value and quality of health economic analyses: implications of utilizing the QHES. Journal of Managed Care Pharmacy. 2003;9(1):53-61.

38. National Institute for Health and Care Excellence. Developing NICE guidelines: the manual Appendix H. London: NICE. 2014.

39. Evers S, Goossens M, de Vet $\mathrm{H}$, van Tulder M, Ament A. Criteria list for assessment of methodological quality of economic evaluations: Consensus on Health Economic Criteria. Int J Technol Assess Health Care. 2005;21(2):240-5.

40. Campbell AJ, Robertson MC, La Grow SJ, Kerse NM, Sanderson GF, Jacobs RJ, et al. Randomised controlled trial of prevention of falls in people aged > or $=75$ with severe visual impairment: the VIP trial. BMJ. 2005;331(7520):817.

41. Cockayne S, Rodgers S, Green L, Fairhurst C, Adamson J, Scantlebury A, et al. Clinical effectiveness and cost-effectiveness of a multifaceted podiatry intervention for falls prevention in older people: a multicentre cohort randomised controlled trial (the REducing Falls with ORthoses and a Multifaceted podiatry intervention trial). Health technology assessment (Winchester, England). 2017;21(24):1-198.

42. Davis JC, Marra CA, Robertson MC, Khan KM, Najafzadeh M, Ashe MC, et al. Economic evaluation of dose-response resistance training in older women: a cost-effectiveness and cost-utility analysis. Osteoporos International. 2011;22(5):1355-66.

43. Davis JC, Marra CA, Robertson MC, Najafzadeh M, Liu-Ambrose T. Sustained economic benefits of resistance training in community-dwelling senior women. Journal of the American Geriatrics Society. 2011;59(7):1232-7.

44. Farag I, Howard K, Hayes AJ, Ferreira ML, Lord SR, Close JT, et al. Cost-effectiveness of a Home-Exercise Program Among Older People After Hospitalization. Journal of the American Medical Directors Association. 2015;16(6):490-6.

45. Farag I, Sherrington C, Hayes A, Canning CG, Lord SR, Close JCT, et al. Economic evaluation of a falls prevention exercise program among people With Parkinson's disease. Movement disorders. 2016;31(1):53-61.

46. Fletcher E, Goodwin VA, Richards SH, Campbell JL, Taylor RS. An exercise intervention to prevent falls in Parkinson's: an economic evaluation. BMC Health Serv Res. 2012;12:426.

47. Hendriks MR, Evers SM, Bleijlevens MH, van Haastregt JC, Crebolder HF, van Eijk JTM. Cost-effectiveness of a multidisciplinary fall prevention program in community-dwelling elderly people: a randomized controlled trial (ISRCTN 64716113). International journal of technology assessment in health care. 2008;24(2):193-202.

48. Irvine L, Conroy SP, Sach T, Gladman JRF, Harwood RH, Kendrick D, et al. Cost-effectiveness of a day hospital falls prevention programme for screened community-dwelling older people at high risk of falls. Age and Ageing. 2010;39(6):710-6.

49. Isaranuwatchai W, Perdrizet J, Markle-Reid M, Hoch JS. Cost-effectiveness analysis of a multifactorial fall prevention intervention in older home care clients at risk for falling. BMC geriatrics. 2017;17(1):199.

50. Jenkyn KB, Hoch JS, Speechley M. How much are we willing to pay to prevent a fall? Cost-effectiveness of a multifactorial falls prevention program for community-dwelling older adults. Canadian Journal on Aging/La Revue canadienne du vieillissement. 2012;31(2):121-37.

51. Kenkre JE, Allan TF, Tobias RS, Parry DJ, Bryan S, Carter YH. Breaking bones, breaking budgets: a clinical and economic evaluation of a prospective, randomized, practice controlled, intervention study in the prevention of accidents in primary care. Family practice. 2002;19(6):675-81.

52. Li F, Harmer P. Economic Evaluation of a Tai Ji Quan Intervention to Reduce Falls in People With Parkinson Disease, Oregon, 2008-2011. Prev Chronic Dis. 2015;12:E120.

53. Patil R, Kolu P, Raitanen J, Valvanne J, Kannus P, Karinkanta S, et al. Cost-effectiveness of vitamin D supplementation and exercise in preventing injurious falls among older home-dwelling women: findings from an RCT. Osteoporosis International. 2016;27(1):193-201.

54. Peeters GMEE, Heymans MW, de Vries OJ, Bouter LM, Lips P, van Tulder MW. Multifactorial evaluation and treatment of persons with a high risk of recurrent falling was not cost-effective. Osteoporosis International. 2011;22(7):2187-96.

55. Polinder S, Boye ND, Mattace-Raso FU, Van der Velde N, Hartholt KA, De Vries OJ, et al. Cost-utility of medication withdrawal in older fallers: results from the improving medication prescribing to reduce risk of FALLs (IMPROveFALL) trial. BMC Geriatr. 2016;16(1):179.

56. Rizzo JA, Baker DI, McAvay G, Tinetti ME. The cost-effectiveness of a multifactorial targeted prevention program for falls among community elderly persons. Medical care. 1996:954-69.

57. Robertson MC, Devlin N, Gardner MM, Campbell AJ. Effectiveness and economic evaluation of a nurse delivered home exercise programme to prevent falls. 1: Randomised controlled trial. BMJ. 2001;322(7288):697-701.

58. Robertson MC, Gardner MM, Devlin N, McGee R, Campbell AJ. Effectiveness and economic evaluation of a nurse delivered home exercise programme to prevent falls. 2: Controlled trial in multiple centres. BMJ. 2001;322(7288):701-4.

Page $22 / 25$ 
59. Robertson MC, Devlin N, Scuffham P, Gardner MM, Buchner DM, Campbell AJ. Economic evaluation of a community based exercise programme to prevent falls. J Epidemiol Community Health. 2001;55(8):600-6.

60. Sach TH, Logan PA, Coupland CA, Gladman JR, Sahota O, Stoner-Hobbs V, et al. Community falls prevention for people who call an emergency ambulance after a fall: an economic evaluation alongside a randomised controlled trial. Age Ageing. 2012;41(5):635-41.

61. Salkeld G, Cumming RG, O'Neill E, Thomas M, Szonyi G, Westbury C. The cost effectiveness of a home hazard reduction program to reduce falls among older persons. Australian and New Zealand journal of public health. 2000;24(3):265-71.

62. Timonen L, Rantanen T, Mäkinen E, Timonen T, Törmäkangas T, Sulkava R. Cost analysis of an exercise program for older women with respect to social welfare and healthcare costs: a pilot study. Scandinavian journal of medicine \& science in sports. 2008;18(6):783-9.

63. Albert SM, Raviotta J, Lin CJ, Edelstein O, Smith KJ. Cost-effectiveness of a statewide falls prevention program in Pennsylvania: Healthy Steps for Older Adults. The American journal of managed care. 2016;22(10):638-44.

64. Beard J, Rowell D, Scott D, van Beurden E, Barnett L, Hughes K, et al. Economic analysis of a community-based falls prevention program. Public Health. 2006;120(8):742-51.

65. Carande-Kulis V, Stevens JA, Florence CS, Beattie BL, Arias I. A cost-benefit analysis of three older adult fall prevention interventions. J Safety Res. 2015;52:65-70.

66. Church J, Goodall S, Norman R, Haas M. An economic evaluation of community and residential aged care falls prevention strategies in NSW. New South Wales public health bulletin. 2011;22(3-4):60-8.

67. Church J, Goodall S, Norman R, Haas M. The cost-effectiveness of falls prevention interventions for older community-dwelling Australians. Aust N Z J Public Health. 2012;36(3):241-8.

68. Day L, Hoareau E, Finch C, Harrison JE, Segal L, Bolton TG, et al. Modelling the impact, cost and benefits of falls prevention measures to support policymakers and program planners. 2009.

69. Farag I, Howard K, Ferreira ML, Sherrington C. Economic modelling of a public health programme for fall prevention. Age Ageing. 2015;44(3):409-14.

70. Frick KD, Kung JY, Parrish JM, Narrett MJ. Evaluating the cost-effectiveness of fall prevention programs that reduce fall-related hip fractures in older adults. J Am Geriatr Soc. 2010;58(1):136-41.

71. Hektoen LF, Aas E, Luras H. Cost-effectiveness in fall prevention for older women. Scand J Public Health. 2009;37(6):584-9.

72. Johansson P, Sadigh S, Tillgren P, Rehnberg C. Non-pharmaceutical prevention of hip fractures - a cost-effectiveness analysis of a community-based elderly safety promotion program in Sweden. Cost effectiveness and resource allocation: C/E. 2008;6:11.

73. Lee RH, Weber T, Colon-Emeric C. Comparison of cost-effectiveness of vitamin D screening with that of universal supplementation in preventing falls in community-dwelling older adults. Journal of the American Geriatrics Society. 2013;61(5):707-14.

74. Ling C, Henderson S, Henderson R, Henderson M, Pedro T, Pang L. Cost benefit considerations of preventing elderly falls through environmental modifications to homes in Hana, Maui. Hawaii medical journal. 2008;67(3):65.

75. Mori T, Crandall C, Ganz DA. Cost-effectiveness of combined oral bisphosphonate therapy and falls prevention exercise for fracture prevention in the USA. Osteoporosis international. 2017;28(2):585-95.

76. Ontario Medical Advisory Secretariat. The Falls/fractures Economic Model in Ontario Residents aged 65 years and over (FEMOR). Ontario health technology assessment series. 2008;8(6):1.

77. Pega F, Kvizhinadze G, Blakely T, Atkinson J, Wilson N. Home safety assessment and modification to reduce injurious falls in community-dwelling older adults: cost-utility and equity analysis. Injury prevention: journal of the International Society for Child and Adolescent Injury Prevention. 2016;22(6):420-6.

78. Poole CD, Smith J, Davies JS. Cost-effectiveness and budget impact of Empirical vitamin D therapy on unintentional falls in older adults in the UK. BMJ open. 2015;5(9):e007910.

79. Sach TH, Foss AJ, Gregson RM, Zaman A, Osborn F, Masud T, et al. Falls and health status in elderly women following first eye cataract surgery: an economic evaluation conducted alongside a randomised controlled trial. Br J Ophthalmol. 2007;91(12):1675-9.

80. Smith RD, Widiatmoko D. The cost-effectiveness of home assessment and modification to reduce falls in the elderly. Australian and New Zealand journal of public health. 1998;22(4):436-40.

81. van der Velde N, Meerding WJ, Looman CW, Pols HA, van der Cammen TJ. Cost effectiveness of withdrawal of fall-risk-increasing drugs in geriatric outpatients. Drugs \& aging. 2008;25(6):521-9.

82. Wu S, Keeler EB, Rubenstein LZ, Maglione MA, Shekelle PG. A cost-effectiveness analysis of a proposed national falls prevention program. Clinics in geriatric medicine. 2010;26(4):751-66.

83. Public Health England. A Return on Investment Tool for the Assessment of Falls Prevention Programmes for Older People Living in the Community. Public Health England. 2018.

84. National Institute for Health and Care Excellence. Guide to the methods of technology appraisal 2013. 2013.

85. Shea BJ, Reeves BC, Wells G, Thuku M, Hamel C, Moran J, et al. AMSTAR 2: a critical appraisal tool for systematic reviews that include randomised or non-randomised studies of healthcare interventions, or both. bmj. 2017;358.

86. Whiting P, Savović J, Higgins JP, Caldwell DM, Reeves BC, Shea B, et al. ROBIS: a new tool to assess risk of bias in systematic reviews was developed. Journal of clinical epidemiology. 2016;69:225-34.

87. Costa S, Cary M, Helling DK, Pereira J, Mateus C. An overview of systematic reviews of economic evaluations of pharmacy-based public health interventions: addressing methodological challenges. Systematic reviews. 2019;8(1):1-20.

Page 23/25 
88. Shiell A. Still waiting for the great leap forward. Health Economics, Policy and Law. 2009;4(2):255-60.

89. Hoomans T, Severens JL, Evers SM, Ament AJ. Value for money in changing clinical practice: should decisions about guidelines and implementation strategies be made sequentially or simultaneously? Medical Decision Making. 2009;29(2):207-16.

90. Hoomans T, Severens JL. Economic evaluation of implementation strategies in health care. BioMed Central; 2014.

91. Markle-Reid M, Dykeman C, Ploeg J, Stradiotto CK, Andrews A, Bonomo S, et al. Collaborative leadership and the implementation of community-based fall prevention initiatives: a multiple case study of public health practice within community groups. BMC health services research. 2017;17(1):141.

92. Olij BF, Erasmus V, Barmentloo LM, Burdorf A, Smilde D, Schoon Y, et al. Evaluation of Implementing a Home-Based Fall Prevention Program among Community-Dwelling Older Adults. Int J Environ Res Public Health. 2019;16(6).

93. Campbell AJ, Robertson MC. Fall prevention: single or multiple interventions? Single interventions for fall prevention. Journal of the American Geriatrics Society. 2013;61(2):281-4.

94. Day LM. Fall prevention programs for community-dwelling older people should primarily target a multifactorial intervention rather than exercise as a single intervention. Journal of the American Geriatrics Society. 2013;61(2):284-5.

95. National Institute for Health and Care Excellence. Supporting investment in public health: Review of methods for assessing cost effectiveness, cost impact and return on investment. London: NICE. 2011.

96. Norheim OF, Baltussen R, Johri M, Chisholm D, Nord E, Brock D, et al. Guidance on priority setting in health care (GPS-Health): the inclusion of equity criteria not captured by cost-effectiveness analysis. Cost Effectiveness and Resource Allocation. 2014;12(1):18.

97. Winser S, Lee SH, Law HS, Leung HY, Bello UM, Kannan P. Economic evaluations of physiotherapy interventions for neurological disorders: A systematic review. Disability and Rehabilitation: An International, Multidisciplinary Journal. 2020;42(7):892-901.

\section{Figures}
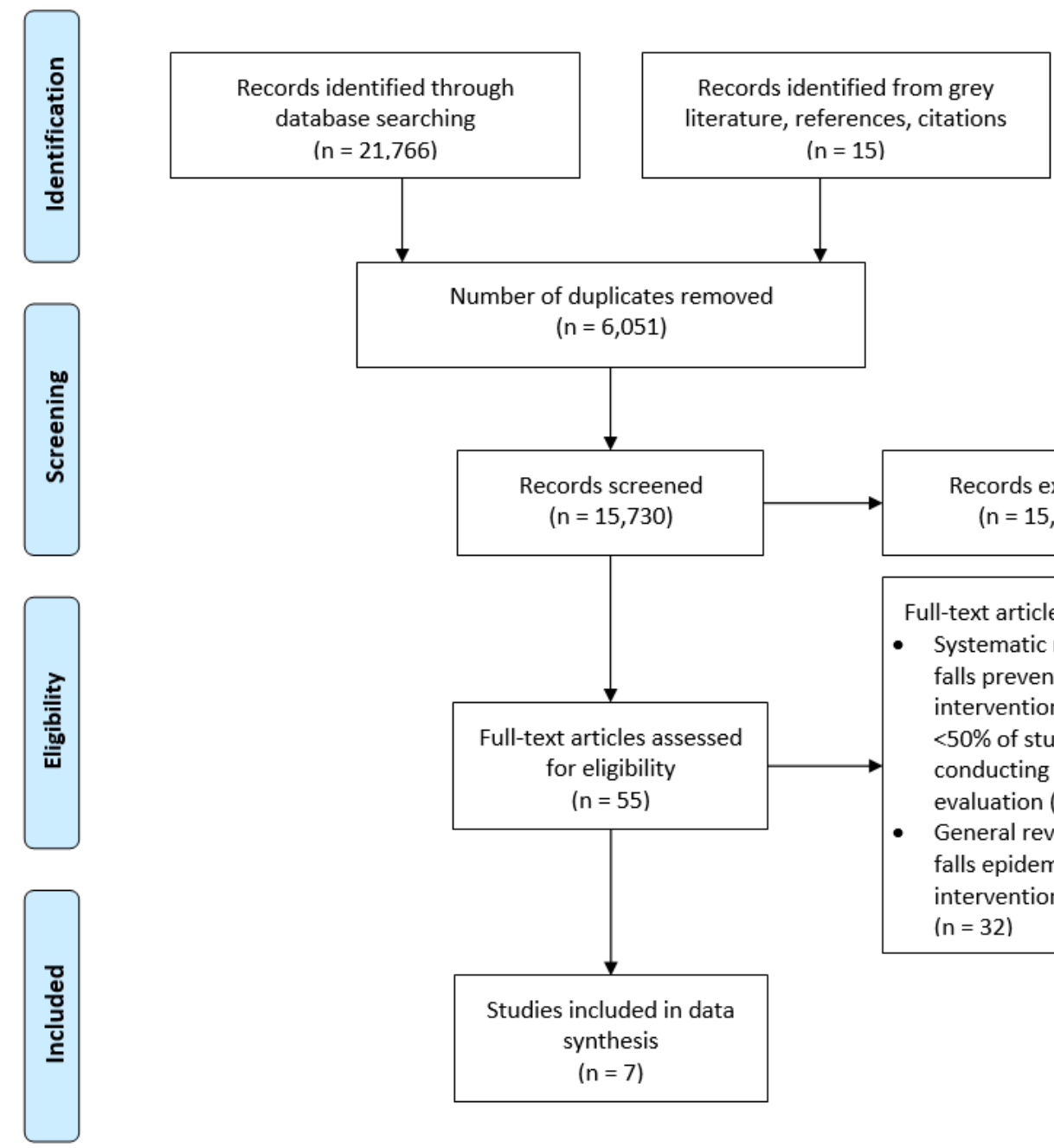

Number of duplicates removed

$(n=6,051)$
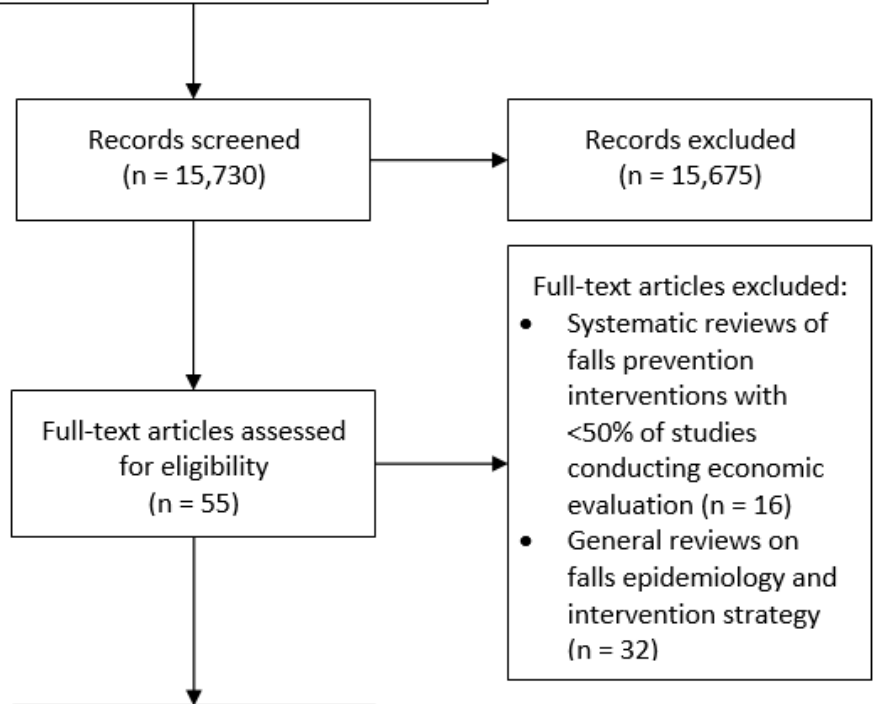

Studies included in data

synthesis

$(n=7)$

Figure 1

Preferred Reporting Items for Systematic Reviews and Meta-Analyses flow diagram. 


\section{Supplementary Files}

This is a list of supplementary files associated with this preprint. Click to download.

- OverviewofsystematicreviewsBMCjournalSupplementarymaterial.docx 